\title{
Numerical Simulations of Single Flow Element in a Nuclear Thermal Thrust Chamber
}

\author{
Gary Cheng, Yasushi Ito ${ }^{\dagger}$, Doug Ross ${ }^{\ddagger}$ \\ Department of Mechanical Engineering \\ University of Alabama at Birmingham, Birmingham, AL 35294-4461 \\ Yen-Sen Chen ${ }^{\S}$ \\ Engineering Sciences, Inc., Huntsville, Alabama, 35815 \\ and \\ Ten-See Wang “ \\ NASA Marshall Space Flight Center, Huntsville, Alabama, 35812
}

\begin{abstract}
The objective of this effort is to develop an efficient and accurate computational methodology to predict both detailed and global thermo-fluid environments of a single flow element in a hypothetical solid-core nuclear thermal thrust chamber assembly. Several numerical and multi-physics thermo-fluid models, such as chemical reactions, turbulence, conjugate heat transfer, porosity, and power generation, were incorporated into an unstructured-grid, pressure-based computational fluid dynamics solver. The numerical simulations of a single flow element provide a detailed thermo-fluid environment for thermal stress estimation and insight for possible occurrence of mid-section corrosion. In addition, detailed conjugate heat transfer simulations were employed to develop the porosity models for efficient pressure drop and thermal load calculations.
\end{abstract}

\section{Introduction}

$\mathrm{T}$ The nuclear thermal rocket is one of the candidate propulsion systems for future space exploration including traveling to Mars and other planets of the solar system. Nuclear thermal propulsion can provide a much higher specific impulse than the best chemical propulsion available today. A basic nuclear propulsion system consists of one or several nuclear reactors that heat hydrogen propellant to high temperatures and then allow the heated hydrogen and its reacting product to flow through a nozzle to produce thrust. In the 1970's, a solid-core design' for the nuclear reactor was developed and tested under a nuclear rocket program called Rover/NERVA. The study showed that the solid-core reactor is a feasible concept to produce specific impulses exceeding $850 \mathrm{sec}$. The solidcore reactor resembles a heat exchanger, which consists of hundreds of heat generating solid flow elements, each flow element containing tens of flow channels through which the hydrogen propellant absorbs heat before entering a nozzle to generate thrust. To achieve maximum efficiency, the reactor often operates at very high temperature and power density. However, the results of Rover/NERVA tests indicated that if the solid fuel temperature exceeds certain values, the flow element may fail due to a phenomenon called mid-section $\operatorname{corrosion}^{1-3}$, which imposes real challenges to the integrity of the flow element. This limit imposes a constraint on the engine performance. The midsection corrosion refers to a crack in the coating layer between the solid fuel and hydrogen flow, whichis designed to protect the solid fuel from chemical attack by the hot hydrogen. Mid-section corrosion can lead to an excessive mass loss of the flow element material in that region. The mid-section corrosion was suspected to be caused by different thermal expansions between the flow element and its coating, where the disparity can be large if strong thermal

\footnotetext{
Associate Professor, Senior Member AIAA, E-mail: gcheng@uab.edu

${ }^{+}$Research Assistant Professor, Member AIAA

${ }^{\ddagger}$ Programmer

${ }^{\S}$ President, Member AIAA

"* Technical Assistant, ER43, Thermal and Combustion Analysis Branch, Senior Member AIAA
} 
gradients occur in that region, and where there is a change of solid thermal property due to irradiation. ${ }^{4}$ Another speculation was that the flow was choked in the long flow channels of the flow element.

It is extremely difficult and expensive to measure the detailed thermal-fluid environment within the entire flow element. The objective of this effort is therefore to develop an efficient and accurate multiphysics thermal-fluid computational methodology to predict environments for a single flow element, similar to those in the Small Engine. ${ }^{1}$ The computational methodology was based on an existing Unstructured-grid Navier-Stokes Internal-external computational fluid dynamics Code $\left(\mathrm{UNIC}^{5-7}\right)$. Conjugate heat transfer $(\mathrm{CHT})$ formulations for coupling fluid dynamics and conductive heat transfer in solids and for flow and conductive heat transfer in porous media were developed and tested. The UNIC code has been well validated and employed to simulate a great variety of engineering problems ranging from internal to external flows, incompressible to compressible flows, single-phase to multi-phase flows, and inert to reacting flows. Two groups of detailed analyses were conducted for a NERVA-type 19-channel flow element, as shown in Figure 1. The first group simulated a full-length 19channel flow element with two different power generation distributions to investigate the occurrence of the mid-section corrosion problem and potential flow chocking in the flow channel. These two distributions include a cosine function in the axial direction with a clipped cosine function in the radial direction, and a clipped cosine function in the axial direction with a clipped cosine function in the radial direction. The second group simulated a one-eighth-length 19-channel flow element with different side-wall temperature and flow channel diameters to obtain data for calibrating the porosity model to be used in the global analysis of the entire thrust chamber. The porosity model is employed to represent the effect of the friction loss and heat transfer as the working fluid flowing through the flow element. The use of the porosity model enables an efficient simulation of the entire thrust chamber and evaluation of its performance during the design cycle.

In order to support both the detailed and global analyses, several physical submodels were either improved or added into the UNIC code ${ }^{8-9}$. These code improvements include an anisotropic drag and heat transfer porosity model to account for the directional effect of the flow channel, a source term in the energy equation to model the power generation of the solid core with user specified distributions, validation of the conjugate heat transfer capability with a well-known thermal analysis code, SINDA ${ }^{10}$. The porosity model development for the flow element and detailed conjugate heat transfer simulations of a powered flow element are reported herein.

\section{Numerical Methodology}

\section{A. Computational Fluid Dynamics}

The employed CFD solver, UNIC, solves a set of Reynolds-averaged governing equations (continuity, NavierStokes, energy, species mass fraction, etc.) to satisfy the conservation laws for a turbulent flow of interest. The set of governing equation can be written in Cartesian tensor form:

$$
\begin{gathered}
\frac{\partial \rho}{\partial t}+\frac{\partial}{\partial x_{j}}\left(\rho V_{j}\right)=0 \\
\frac{\partial\left(\rho V_{i}\right)}{\partial t}+\frac{\partial}{\partial x_{j}}\left(\rho V_{j} V_{i}\right)=-\frac{\partial p}{\partial x_{i}}+\frac{\partial \tau_{j i}}{\partial x_{j}}+S_{V} \\
\frac{\partial\left(\rho h_{t}\right)}{\partial t}+\frac{\partial}{\partial x_{j}}\left(\rho V_{j} h_{t}\right)=\frac{\partial p}{\partial t}+\frac{\partial}{\partial x_{j}}\left[\left(\frac{\mu}{\operatorname{Pr}}+\frac{\mu_{t}}{\operatorname{Pr}_{t}}\right) \frac{\partial h}{\partial x_{j}}\right]+\frac{\partial V_{i} \tau_{j i}}{\partial x_{j}}+Q_{r}+S_{h} \\
\frac{\partial(\rho k)}{\partial t}+\frac{\partial}{\partial x_{j}}\left(\rho V_{j} k\right)=\frac{\partial}{\partial x_{j}}\left[\left(\mu+\frac{\mu_{t}}{\sigma_{k}}\right) \frac{\partial k}{\partial x_{j}}\right]+\rho\left(P_{k}-\varepsilon\right)
\end{gathered}
$$




$$
\begin{gathered}
\frac{\partial(\rho \varepsilon)}{\partial t}+\frac{\partial}{\partial x_{j}}\left(\rho V_{j} \varepsilon\right)=\frac{\partial}{\partial x_{j}}\left[\left(\mu+\frac{\mu_{t}}{\sigma_{\varepsilon}}\right) \frac{\partial \varepsilon}{\partial x_{j}}\right]+\rho \frac{\varepsilon}{k}\left(C_{1} P_{k}-C_{2} \varepsilon+C_{3} \frac{P_{k}^{2}}{\varepsilon}\right) \\
\frac{\partial\left(\rho \alpha_{i}\right)}{\partial t}+\frac{\partial}{\partial x_{j}}\left(\rho V_{j} \alpha_{i}\right)=\frac{\partial}{\partial x_{j}}\left[\left(\frac{\mu}{S c}+\frac{\mu_{t}}{S c_{t}}\right) \frac{\partial \alpha_{i}}{\partial x_{j}}\right]+S_{i} \\
\tau_{j i}=\left(\mu+\mu_{t}\right)\left(\frac{\partial V_{i}}{\partial x_{j}}+\frac{\partial V_{j}}{\partial x_{i}}-\frac{2}{3} \frac{\partial V_{l}}{\partial x_{l}} \delta_{i j}\right)-\frac{2}{3} \rho k \delta_{i j} ; \quad P_{k}=\mu_{t}\left(\frac{\partial V_{i}}{\partial x_{j}}+\frac{\partial V_{j}}{\partial x_{i}}-\frac{2}{3} \frac{\partial V_{l}}{\partial x_{l}} \delta_{i j}\right) \frac{\partial V_{i}}{\partial x_{j}}
\end{gathered}
$$

where $\rho$ is the fluid density $p$ is the pressure, $V_{j}=(u, v, w)$ stands for the velocity components in $x$-, $y$-, and $z$ coordinates respectively, $h_{t}$ and $h$ are the total and static enthalpies, $k$ is the turbulence kinetic energy, $P_{k}$ and $\varepsilon$ are the production and dissipation rates of turbulence, $\alpha_{i}$ and $S_{i}$ are the mass fraction and production/destruction rate of $i$-th species, $Q_{r}$ is the radiative heat flux, $S_{V}$ and $S_{h}$ are the source/sink terms of the momentum and energy equations, $\mu$ and $\mu_{t}$ are the fluid and eddy viscosity, $\tau_{j i}$ represents the sum of the viscous and Reynolds stresses, $\operatorname{Pr}$ and $\operatorname{Pr}_{t}$ are the Prandtl and turbulent Prandtl numbers, $S c$ and $S c_{t}$ are Schmidt and turbulent Schmidt numbers, $C_{1}, C_{2}, C_{3}, \sigma_{k}$, and $\sigma_{\varepsilon}$ are turbulence modeling constants. Detailed expressions for the $k-\varepsilon$ models and wall functions can be found in Ref. 11. An extended $k-\varepsilon$ turbulence mode ${ }^{12}$ was used to describe the turbulent flow. A modified wall function approach $^{13,14}$ was employed to provide wall boundary layer solutions that are less sensitive to the near-wall grid spacing.

A predictor and multi-corrector pressure-based solution algorithm ${ }^{15,}{ }^{16}$ was employed in the UNIC code to couple the set of governing equations such that both compressible and incompressible flows can be solved in a unified framework without using ad-hoc artificial compressibility and/or a pre-conditioning method. The employed predictor-corrector solution method ${ }^{5}$ is based on modified pressure-velocity coupling approach of the SIMPLEtype $^{16}$ algorithm which includes the compressibility effects and is applicable to flows at all speeds. In order to handle problems with complex geometries, the UNIC code employs a cell-centered unstructured finite volume method $^{6,7}$ to solve for the governing equations in the curvilinear coordinates, in which the primary variables are the Cartesian velocity components, pressure, total enthalpy, turbulence kinetic energy, turbulence dissipation and mass fractions of chemical species.

The inviscid flux is evaluated through the values at the upwind cell and a linear reconstruction procedure to achieve second order accuracy. A multi-dimensional linear reconstruction approach by Barth and Jespersen ${ }^{17}$ was used in the cell reconstruction to achieve higher-order accuracy for the convection terms. A second-order centraldifference scheme was employed to discretize the diffusion fluxes and source terms. A dual-time sub-iteration method is employed for time-accurate time-marching computations. A pressure damping term, Rhie and Chow ${ }^{18}$, is applied to the evaluation of mass flux at the cell interface to avoid the even-odd decoupling of velocity and pressure fields. All the discretized governing equations are solved using the preconditioned Bi-CGSTAB ${ }^{19}$ matrix solver, except the pressure-correction equation which has an option to be solved using GMRES ${ }^{20}$ matrix solver when the matrix is ill-conditioned. An algebraic multi-grid (AMG) solver ${ }^{21}$ was included such that users can activate to improve the convergence if desired. In order to efficiently simulate problems involving large numbers of meshes, the UNIC code employed parallel computing with domain decomposition, where exchange of data between processors is done by using MPI ${ }^{22}$. Domain decomposition (partitioning the computational domain into several subdomains handled by different computer processors) can be accomplished by using METIS $^{23}$ or a native partitioning routine in the UNIC code.

\section{B. Conjugate Heat Transfer (CHT):}

The framework of CHT is to solve the heat transfer in the fluid flow and the heat conduction in the solid in a coupled manner. The governing equation describing the heat transfer in the fluid flow is shown in Eq. (3), where the heat conduction in the solid can be written as:

$$
\frac{\partial \rho C_{p} T}{\partial t}-\frac{\partial}{\partial x_{i}}\left(\kappa \frac{\partial T}{\partial x_{i}}\right)=Q_{v}+\frac{\partial Q_{s}}{\partial x_{i}}
$$


where $Q_{v}$ and $Q_{s}$ represent source terms from volumetric and boundary contributions, respectively. $\kappa$ and $C_{p}$ denote the thermal conductivity and capacity of the solid material, respectively. In the case of simulating solid core with power generation, $Q_{v}$ depends on power generation distributions function employed. In the present study, three power distribution functions were examined: (i) pure sine function in the axial direction with pure cosine function in the radial direction; (ii) pure sine function in the axial direction with clipped cosine function in the radial direction; (iii) Clipped sine function in the axial direction with clipped cosine function in the radial direction. The temperature value at the fluid-solid interface is obtained by enforcing the heat flux continuity condition, i.e. $Q_{s}=-q_{w}$ where $q_{w}$ is the heat flux from the fluid to the solid calculated by solving Eq. (3) including the turbulence effect if applicable. In order to achieve numerical stability and enforce the heat flux continuity condition, an implicit treatment of the temperature at the fluid-solid interface is employed. In this approach, Eq. (8) can be discretized as

$$
\frac{1}{2} \rho C_{p} \frac{T_{b}^{n+1}-T_{b}^{n}}{\Delta t}=\frac{1}{\Delta y_{n}}\left(k \frac{T_{c}^{n}-T_{b}^{n}}{\Delta y_{n}}-q_{w}\right)
$$

where the superscripts $n+1$ and $n$ denote the values at the next and current time levels, respectively. $T_{b}$ and $T_{c}$ are the temperatures at the fluid-solid interface and at the center of the solid cell next to the fluid-solid interface in respect. $y_{n}$ represents the normal distance from the interface to the center of the solid cell next to the fluid-solid interface. The $1 / 2$ factor on the left hand side of the above equation is because only half of the solid cell is involved in the control volume. It can be seen that this scheme can be applied to both transient and steady state simulations. For steady-state simulations, an acceleration factor can be used to improve convergence of heat conduction in the solid. Implementation of the implicit treatment has been validated by comparing with the SINDA code.

\section{Porosity Model:}

In the present study, a porosity model was developed to represent the momentum and energy transport through an assembly of flow pipes and the heat conduction through the solid material within a flow element. Hence, the porosity model will include separate temperatures and thermal conductivities for both the solid material and fluid flow. The momentum and energy equations for the fluid flow are the same as Eqs. 2 \& 3, except the source terms will be modified to account for the extra friction loss and heat transfer. The source term of the momentum equation (Eq. 2) can be expressed as

$$
\vec{S}_{V}=\frac{D}{\xi \forall}=\frac{1}{2} \frac{\rho\left|V_{s}\right| C_{D}}{\xi^{2} d / 4} \vec{V}_{s} \text { and } C_{D}=f_{D} C_{f}
$$

where $D$ is the drag force modeled by the porosity model, $\xi$ is the porosity factor for the porous region, $V_{s}$ is the superficial flow velocity through the porous region, $d$ is diameter of the flow channel, $\forall$ is the total volume of the porous region, $C_{D}$ is the drag coefficient, and $f_{D}$ is a modeling constant that will be tuned for the geometry of interest in the present study by comparing solutions of the porosity model and the detailed CHT model. An empirical correlation of the friction factor $\left(C_{f}\right)$ for the flow through a pipe, known as the Blasius formula ${ }^{24}$, is used and expressed as follows.

$$
C_{f}=0.0791 \mathrm{Re}_{d}^{-0.25} \quad \text { where } \quad \operatorname{Re}_{d}=\frac{\rho V_{s} d}{\mu \xi}
$$

The source term for the energy equation (Eq. 3) can be calculated as

$$
S_{h}=\frac{Q}{\xi \forall}=\frac{f_{h}}{2} \frac{\rho\left|V_{s}\right| C_{f}}{\operatorname{Pr}^{2 / 3} \xi d / 4} C_{p} \Delta T \quad \text { where } \quad \Delta T=T_{s}-T_{g}
$$

where $Q$ is the heat sink/source due to the fluid-solid interaction in the porous region, $\mathrm{Pr}$ is the Prandtl number of the fluid, $f_{h}$ is aa modeling constant will be tuned by comparing solutions of the porosity model and the detailed CHT model, and $T_{s}$ and $T_{\mathrm{g}}$ are the temperatures of the solid and fluid at the same location, respectively. In addition, the source term of the heat conduction equation (Eq. 8) in the porous region can be calculated as 


$$
Q_{v}=\frac{Q}{(1-\xi) \forall}=\frac{f_{h}}{2} \frac{\rho\left|V_{s}\right| C_{f}}{\operatorname{Pr}^{2 / 3}(1-\xi) d / 4} C_{p} \Delta T \quad \text { and } \quad \Delta T=T_{g}-T_{s}
$$

while $Q_{s}=0$.

\section{Numerical Meshes}

For the numerical simulations with the CHT model, two mesh systems need to be constructed. One of them is employed to model the entire computational domain including both the solid grain and the flow channel. The other one is used by the code to identify the region of solid material, and thus it can be very coarse. Hence, there is no need for a special grid generator to construct multi-block meshes for separating the solid and the fluid region, nor the identification for the fluid-solid interface. In the present study, various flow element geometries were simulated, such as different flow channel diameter, with and without coating layer in the solid core, and different flow channel length. Hence, a geometry/grid template, as shown in Figure 2, was developed based on MiniCAD ${ }^{25-27}$ to expedite the geometry and grid generation process by allowing the user to interactively change 1) the ratio of the gap between flow channels to the diameter of flow channel, 2) the ratio of the size of the flow element to the diameter of the flow channel, 3) thickness of the coating layer, and 4) the length of the flow element. The benefit of using MiniCAD is the ability to have graphical feedback while building the geometry and grid, and to easily move dimensional values from the geometry into the template to make it easy to create grids with modified values. In the present study, a $60^{\circ}$ pi-section of a single flow element, which includes 3 and 1/6 flow channels, was simulated.

MiniCAD is a graphical user interface (GUI) built on the Geometry Grid Toolkit (GGTK). The geometry that defines the dimensions of the solid grain was also exported as a discrete surface, which can be used by an in-house grid generator to construct the hybrid mesh for the entire flow element. The process of building grid in MiniCAD involves three basic steps. Step one is to create or import the geometry. Step two is to pass the geometry to a grid topology to ensure that the mesh built will be geometrically watertight. Step three is to build the mesh based on the grid topology. Based on our investigation, we found the most efficient structured grid topology to model the solid grain is an O-type grid. In this case, the solid grain was decomposed into multiple blocks ( 5 blocks for cases without a coating layer, and 10 blocks for those with a coating layer), where the O-grids of each block were used to model the solid grain and coating layer (if present) surrounding each flow channel as shown in Figure 2. A separate block is needed to identify the coating layer because it has different thermal properties from those of the solid grain. The boundary of the O-grid geometry for each flow channel was built as a planar trimmed surface. This geometry was converted to the topology structure, and then a 2-D grid was built on the faces. Since the grid for the solid grain is only used to identify the region of solid grain and is geometrically similar in the axial direction, it was only necessary to create the 3-D grid by extruding the 2-D grid in the axial direction, which can be seen in Figure 3.

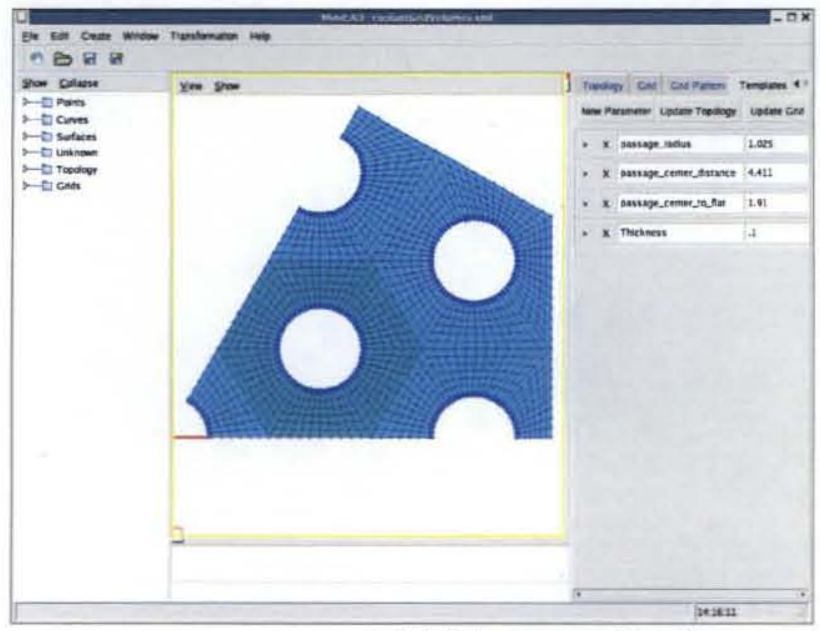

(a) $0.1 \mathrm{~mm}$ coating layer

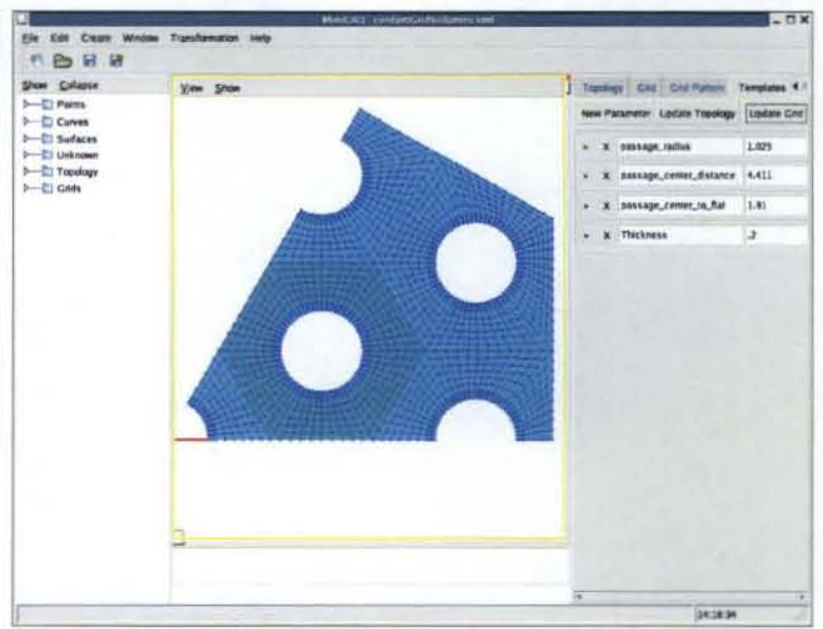

(b) $0.2 \mathrm{~mm}$ coating layer

Figure 2. Layout of the developed geometry/grid template (flow channel diameter $=\mathbf{2 . 0 5} \mathbf{m m}$ ) 


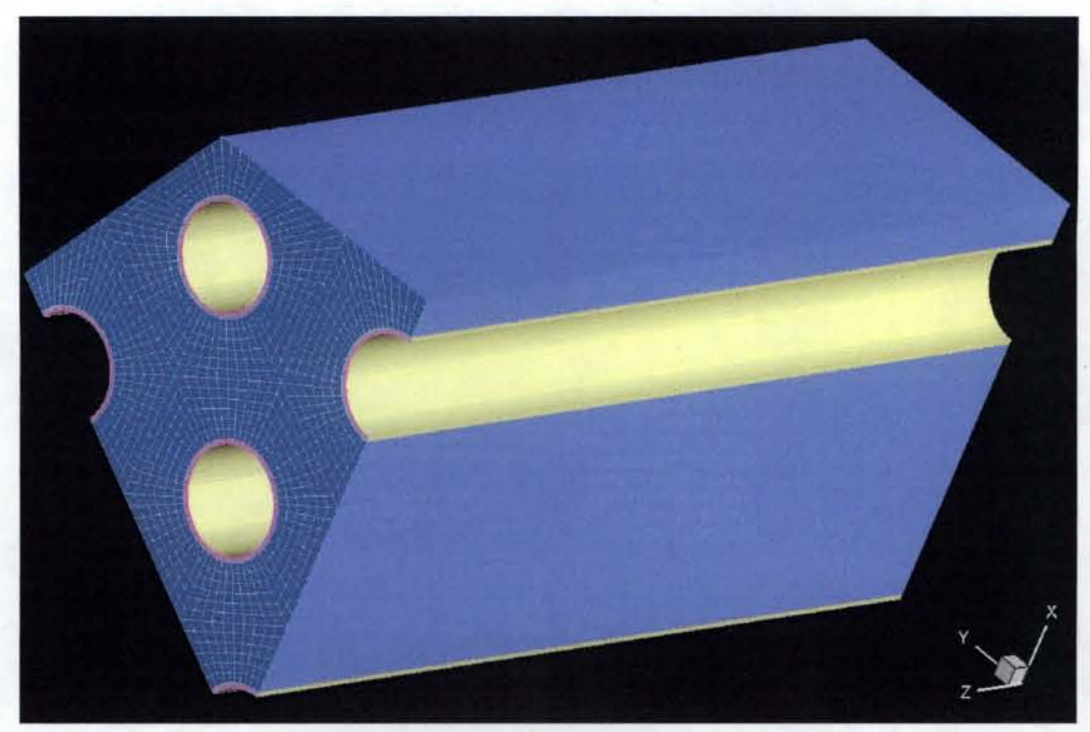

Figure 3. Structured grid system for the solid grain and coating layer of a flow element

To accurately resolve the momentum and energy transport near the solid-fluid interface, a hybrid mesh topology was employed to generate the mesh for the entire flow element. The procedure used by our in-house grid generator is to construct the surface mesh before creating the 3-D volume mesh. For surface mesh generation, a direct advancing front method is used ${ }^{0,0}$. A discrete model imported from MiniCAD is used as a background mesh, on which a new surface mesh suitable for computational simulations is created. Geometrical features are extracted based on a folding angle at each edge. A user specifies node distributions on the geometrical features, which form an initial front for the advancing front method. The surface triangulation is performed in the physical 3D space in order to check the quality of triangles easily.

For volume mesh generation, two approaches were used. One is a method to create regular hybrid meshes based on an advancing layer method. In this method, a multiple marching direction approach is employed to create better control volumes around sharp convex corners ${ }^{0}$. Figure 4 shows a hybrid mesh inside a flow element, which has 335,207 nodes, 688,027 tetrahedra, 21,476 prisms, 33,655 pyramids and 167,259 hexahedra. Quadrangles are used for the pipe surfaces. Multiple marching directions are defined at pipe inlets, which enable smooth transition of the mesh size there.
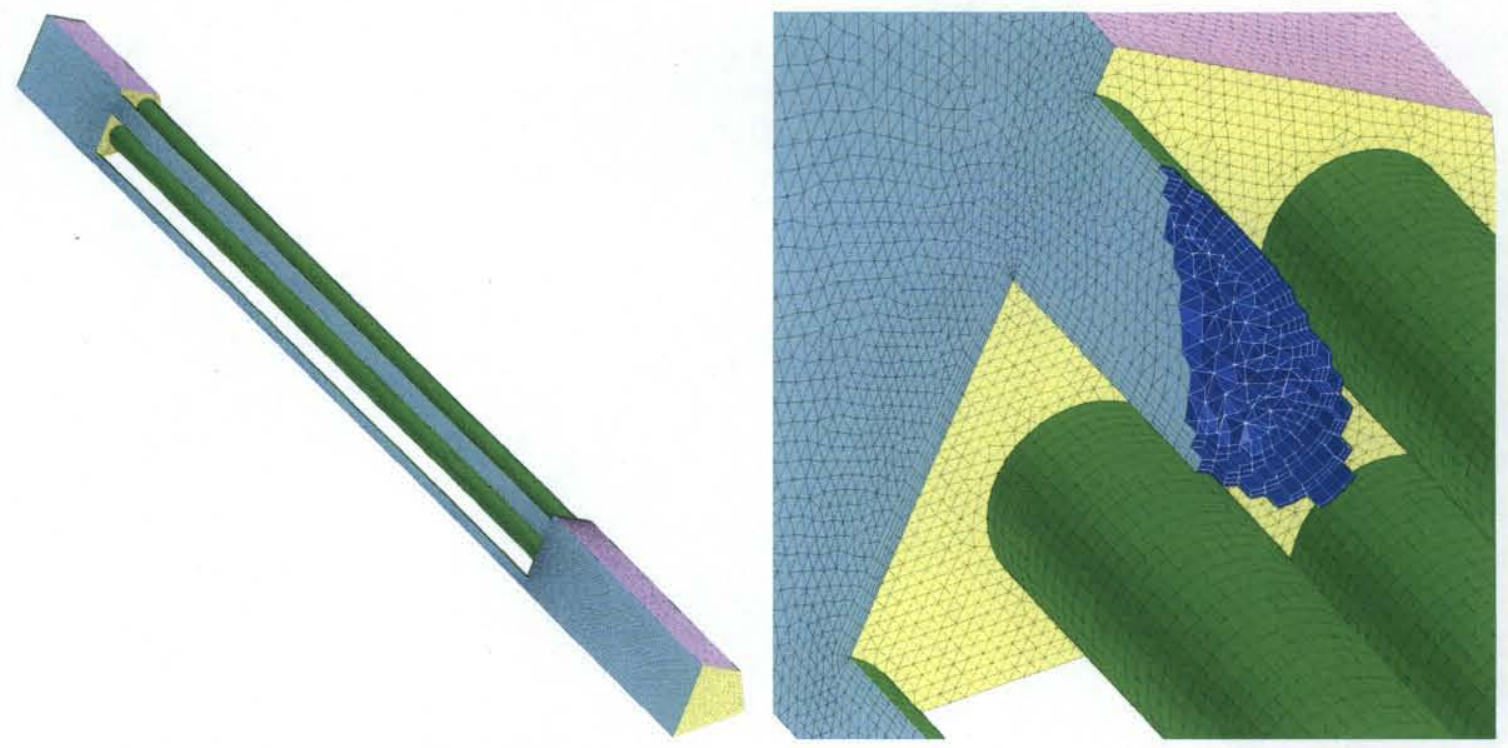

Figure 4. Regular hybrid meshes: (a) surface mesh; (b) cross-section of hybrid volume mesh 
The other volume mesh generation method is an extrusion method based on a 2-D mesh to create volume meshes more easily. Every cross-section across the symmetry axis is the same. First, a 2-D mesh is created as shown in Figure 5. Second, it is extruded toward the symmetry axis, following the user-specified spacing. Third, boundary surfaces are numbered so that boundary conditions are set for CFD simulations.

\section{Results and Discussion}

\section{A. Calibration of porosity model:}

To tune the modeling constant employed in the porosity model, numerical experiments of simulating coolant flow through short-length flow elements with fixed boundary wall temperature $\left(T_{w}\right)$ using the CHT model were conducted to generate the anchoring data. The computational domain and

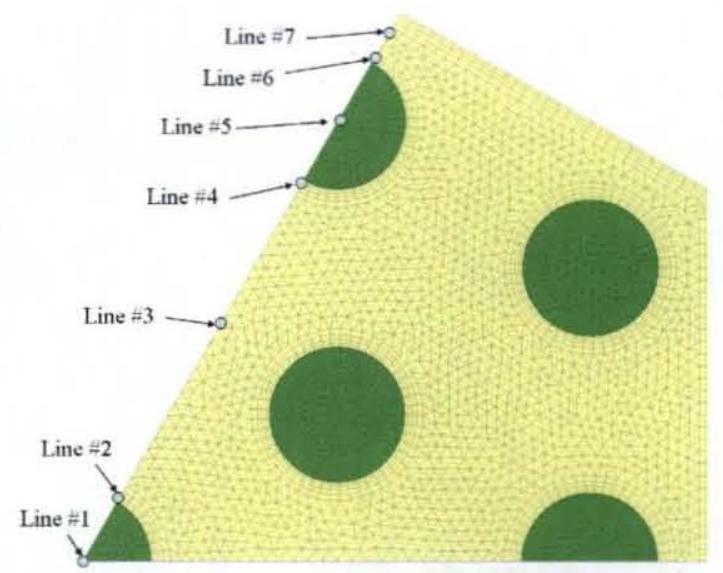

Figure 5. 2-D mesh for the extrusion method: solid part (yellow) and fluid part (green) boundary setup is shown in Figure 6. In this numerical experiment, the flow element has a width of $19.1 \mathrm{~mm}$, length of $110 \mathrm{~mm}$, and the inlet flow conditions are the same as those in Table 1, except the pressure is 34 atm. Even though the flow element was shortened from $890 \mathrm{~mm}$ to $110 \mathrm{~mm}$ to save the computational time, a length-diameter ratio $\left(L_{p} / D_{p}\right)$ of 55 is enough to obtain a fully developed flow result for anchoring the porosity model. Two groups of numerical experiments were conducted. There are three cases in the first group, where the flow channel diameter is $2.05 \mathrm{~mm}$ and the wall temperatures are set to be $3000 \mathrm{~K}, 2000 \mathrm{~K}$, and $1000 \mathrm{~K}$, respectively. In the second group, the wall temperature was set to be $3000 \mathrm{~K}$, while three flow channel diameters $(2.05 \mathrm{~mm}, 2.29 \mathrm{~mm}$, and $2.54 \mathrm{~mm}$ ) were modeled. A hybrid mesh system of 1.8 millions cells was used to simulate the coolant flow through a flow element using CHT.

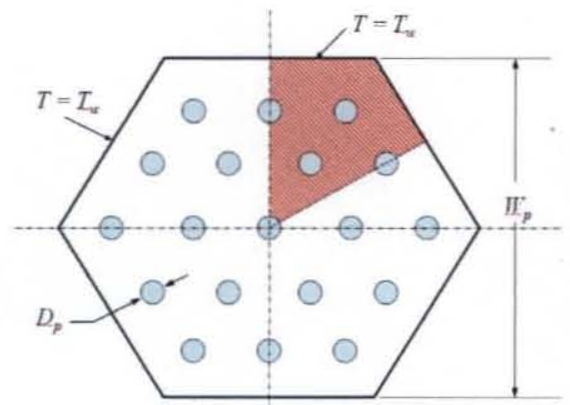

(a) Cross-sectional view

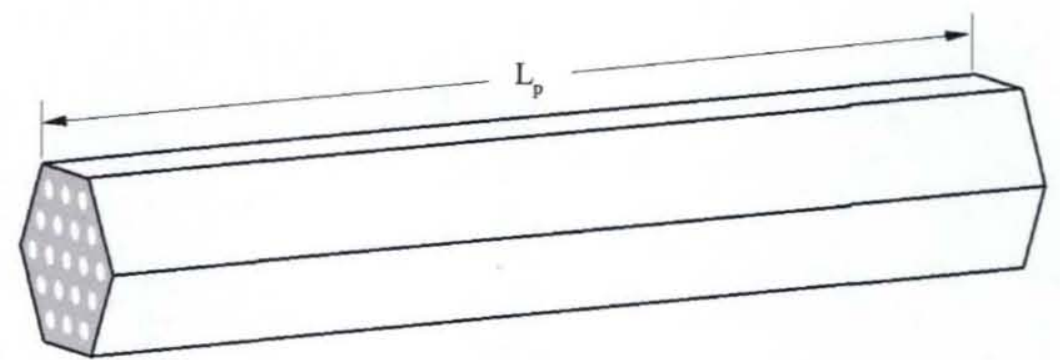

(b) Side view

Figure 6. Sketch of the flow element geometry and its boundary conditions

The numerical results of the first group (varying wall temperatures) and second group (varying flow channel diameter) follow the similarity rule, and thus only the flowfield of the case with a wall temperature of $3000 \mathrm{~K}$ and a flow channel diameter of $2.05 \mathrm{~mm}$ is plotted as shown in Figures 7-9. Figure 7 shows the pressure and temperature distributions at each boundary including the fluid-solid interface. The streamlines and velocity vectors of the fluid flow near the entrance and exit of the flow element are illustrated in Figure 8. Figure 9 shows the pressure distributions along the centerlines of the center and outer flow channels, and temperature distributions along 4 axial lines (Line \#1: centerlines of the center flow channel; Line \#3: the line between the center and outer flow channels; Line \#5: centerlines of the outer flow channel; Line \#7: the line between the outer flow channel and the edge of the flow element). As noted in Figure 9, a portion of temperatures along Lines \#3 and \#7 are the temperatures of the solid grain instead of the fluid flow. The temperature rises and pressure drops calculated from the numerical experiments will be used to tune the porosity model, and thus the results of other cases will be shown in the following comparison with the results of the porosity model. 

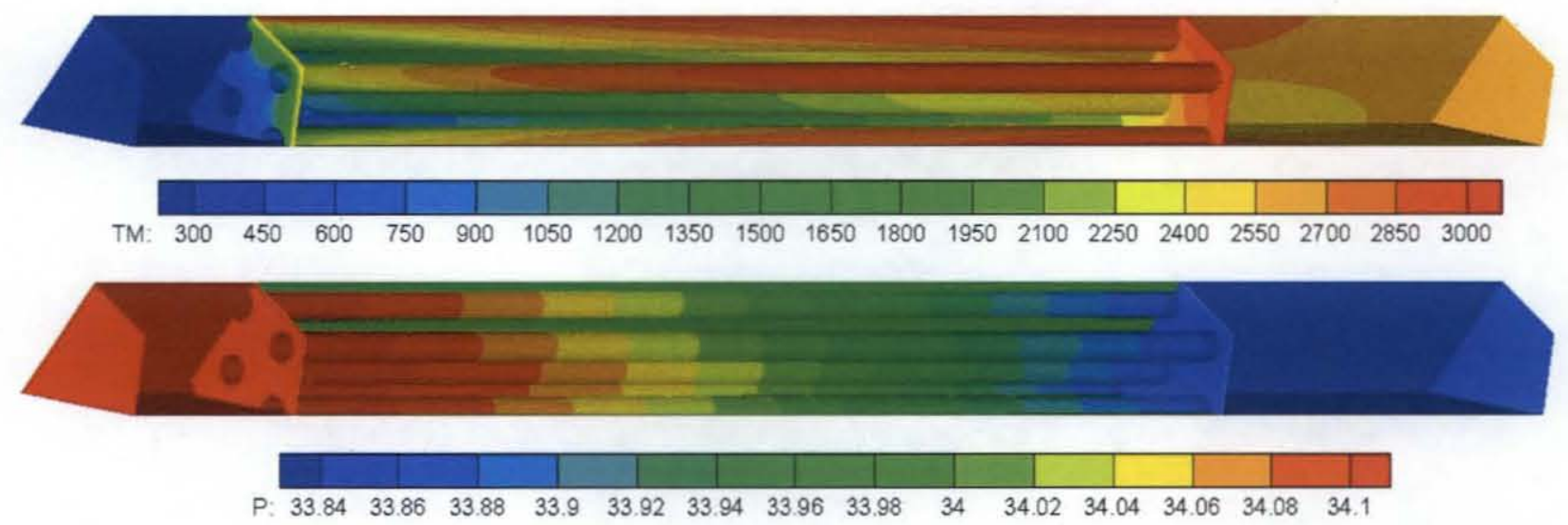

Figure 7. Temperature (top) and pressure (bottom) distributions for flow through a heated flow element with CHT $\left(T_{w}=3000 \mathrm{~K}, D_{p}=2.05 \mathrm{~mm}\right)$
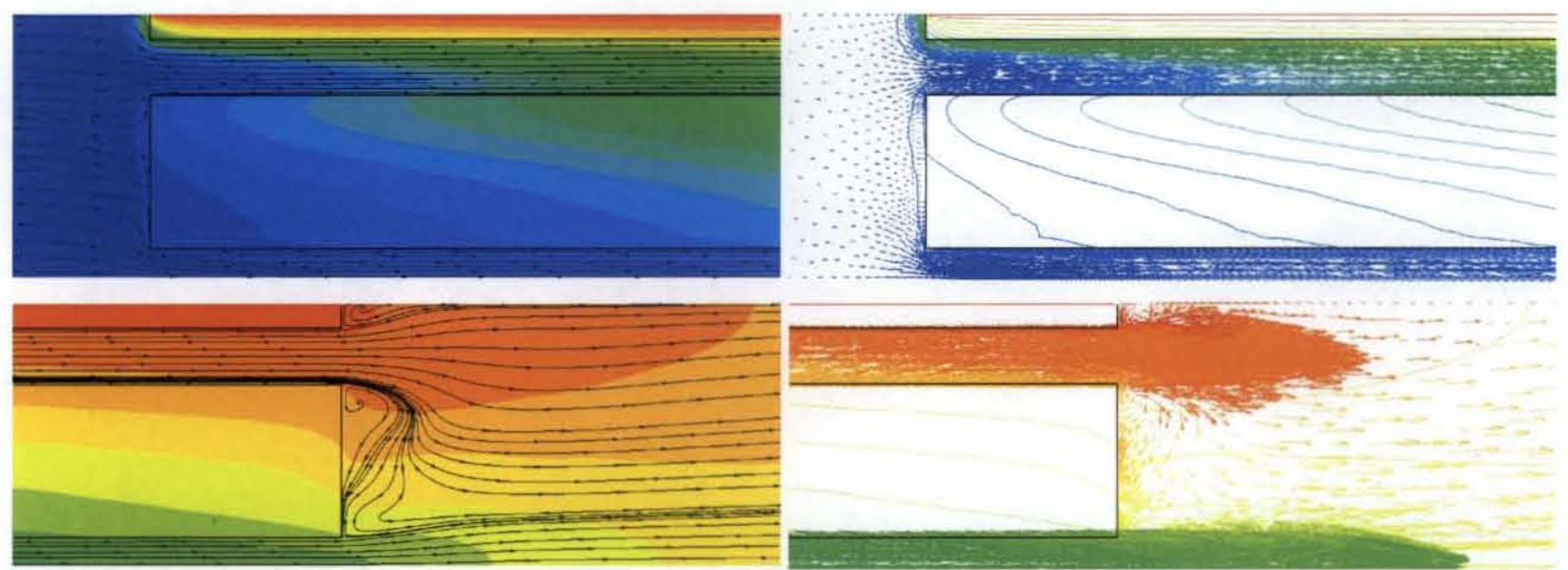

Figure 8. Streamlines (left) and velocity vectors (right) near the entrance (top) and exit (bottom) of the flow element $\left(T_{w}=3000 \mathrm{~K}, D_{p}=2.05 \mathrm{~mm}\right)$
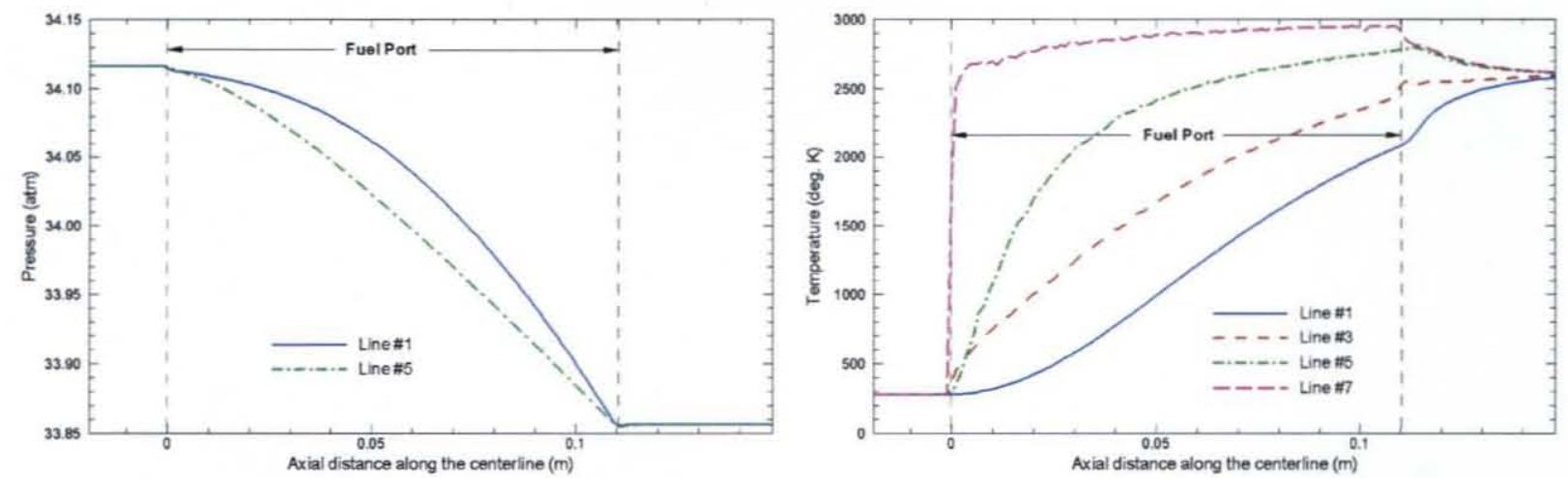

Figure 9. Pressure (left) and temperature (right) distributions along the axial direction $\left(T_{w}=3000 \mathrm{~K}, D_{p}=\right.$ $2.05 \mathrm{~mm}$ )

The cases simulated in the previous numerical experiments using CHT repeated using the porosity model. A hybrid mesh system with $23 \mathrm{k}$ cells was used to model the flow element, which is much coarser than that used in the simulation with CHT. The modeling constants of the employed porosity model, shown in Eqs 10 and 11, were tuned based on the cases of $T_{w}=3000 \mathrm{~K}$ and $D_{p}=2.05 \mathrm{~mm}$, and were selected to be $f_{D}=18$, and $f_{h}=0.22$. The selection 
of $T_{w}=3000 \mathrm{~K}$ is because it is closest to the temperature calculated in the simulation of the full-length flow element with power generation and CHT. The calculated temperature and pressure distributions of this case are plotted as shown in Figure 10, which shows close resemblance to the result shown in Figure 7. With the same modeling constants, other cases were simulated with the porosity model. The results are compared to those obtained from the simulations using CHT, and are shown in Figures 11 and 12. Figure 11 indicates that the effect of the boundary temperature on pressure gradients calculated by the CHT model is more pronounced than that calculated by the porosity model. However, temperature distributions predicted by the porosity model agree very well with those by the CHT model. Variation of pressure gradients for different flow channel sizes calculated by the CHT and porosity models show minor discrepancy as exhibited in Figure 12. On the contrary, the result of the porosity model displays a stronger influence of the flow channel size on temperature distributions than that by the CHT model. The cause for the discrepancy between the porosity and CHT models may be attributed to several factors. Since the local heat transfer and drag coefficients are highly dependent on the local flow Reynolds numbers, the value of the fluid viscosity need to be accurately accounted for. In the present study, the fluid viscosity of air, instead of hydrogen, was used, which can be one of the causes of the discrepancy. The accuracy of the correlation between the fluid viscosity and temperature employed in the code also needs to be further examined. The Blasius formula for the friction loss coefficient, developed based on the isothermal flow, may cause some errors of the non-isothermal fluid flow in the present study, and thus requires further refinement. The result shows the need for further investigation of the employed empirical correlations of the porosity model such that it can be applied to a wider variation of flow conditions and flow element geometries. Despite this discrepancy, if the operating conditions and geometry are close to the tuning conditions (maximum $T_{w}=3000 \mathrm{~K}, D_{p}=2.05 \mathrm{~mm}$ ), it is appropriate to use the tuned porosity model to predict the global performance of nuclear thermal thrust chamber assembly.

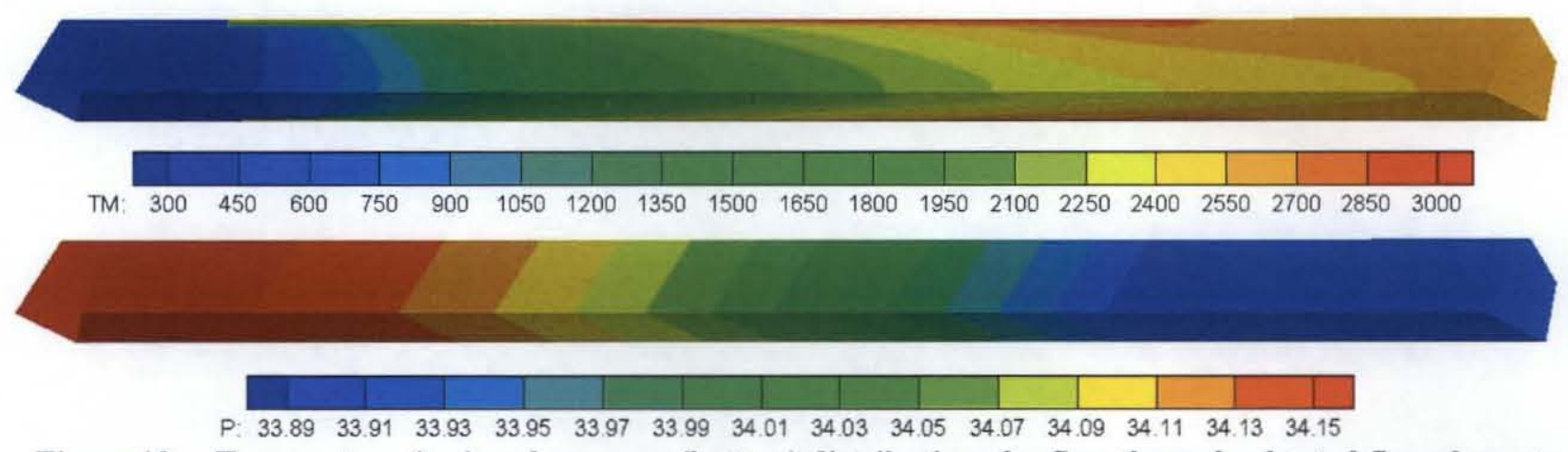

Figure 10. Temperature (top) and pressure (bottom) distributions for flow through a heated flow element with porosity model $\left(T_{w}=3000 \mathrm{~K}, D_{p}=2.05 \mathrm{~mm}\right)$
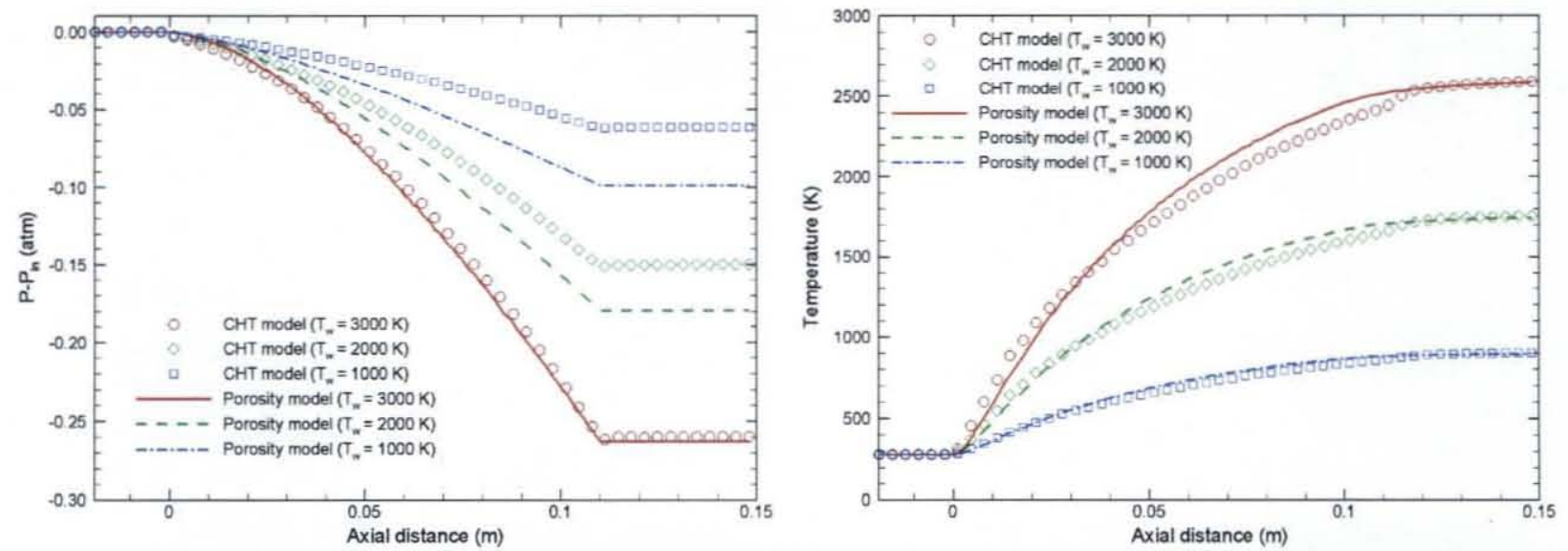

Figure 11. Comparisons of pressure gradient (left) and temperature (right) distributions with various wall temperatures $\left(D_{p}=2.05 \mathrm{~mm}\right)$ 

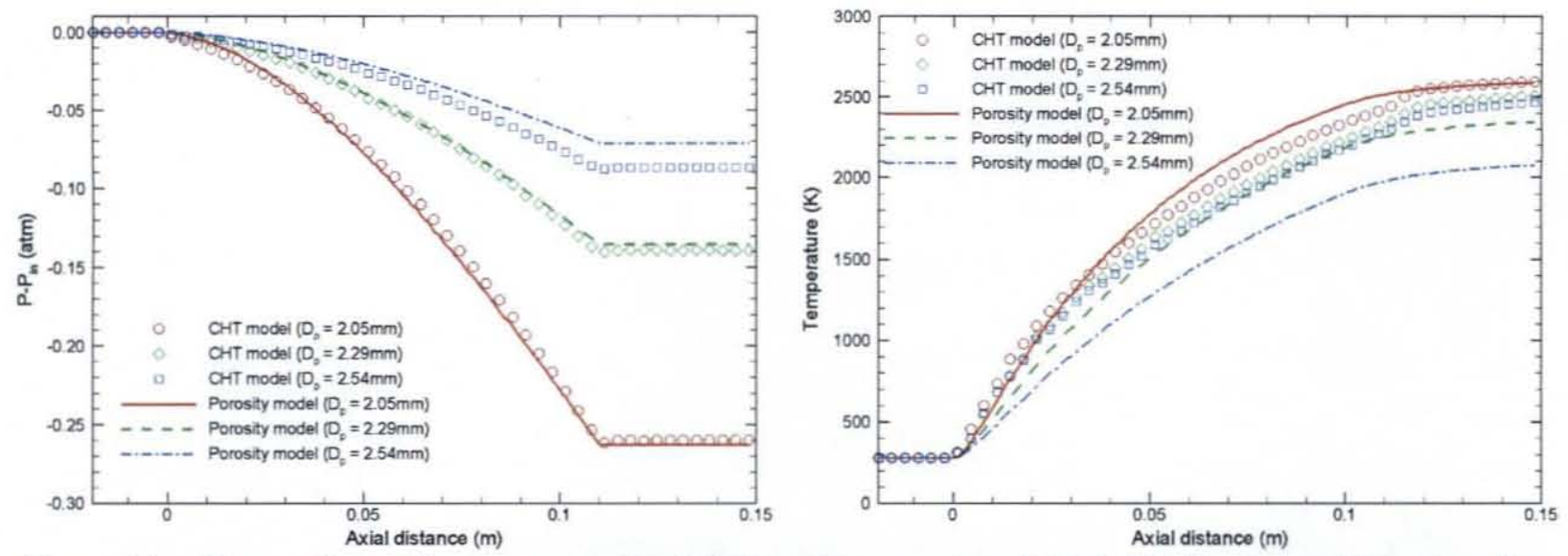

Figure 12. Comparisons of pressure gradient (left) and temperature (right) distributions with various flow channel diameters $\left(T_{w}=\mathbf{3 0 0 0} \mathrm{K}\right)$

\section{B. Full-length single flow element with power generation:}

To investigate the mid-section corrosion problem, a coolant flow (gaseous hydrogen) though the full-length, innermost flow element (the largest energy output) with a given power generation source was simulated to understand the thermal and fluid environment with the flow element. The layout of the single flow element simulated and its geometric definitions are similar to those illustrated in Figure 6, where only 1/6 of the flow element (shaded area in Figure 6) is considered in the numerical simulation due to geometrical symmetry. The only difference is that instead of an isothermal condition (at $T_{w}$ ), an adiabatic boundary condition was imposed at the edge of the flow element. A hybrid mesh system, as shown in Figures 4-5, with 4.5 millions cells was used to model the flow element, the upstream and downstream sections. The values of the geometric dimension of the flow element, and the inflow conditions were obtained from Ref. 31, and are listed in Table 1. A coating layer ( $\mathrm{ZrC}$ composite) with a thickness of $0.127 \mathrm{~mm}$ was added to each flow channels. In the numerical simulation, the thermal properties of the flow element (UC-C-ZrC composite) and the coating layer ( $\mathrm{ZrC}$ composite) vary with temperature and can be found in Ref. 2, where the values of those properties at a temperature of $300 \mathrm{~K}$ are listed in Table 2 as an example. The thermal properties (UC-C-ZrC composite ${ }^{2}$ ) are listed in Table 2. It is noted that in the actual simulation the thermal properties of $\mathrm{UC}-\mathrm{C}-\mathrm{ZrC}$ and $\mathrm{ZrC}$ composites vary with temperature, where the values listed in Table 2 are the properties at a temperature of $300 \mathrm{~K}$. An entrance section of 1 element width $\left(W_{p}\right)$ was added to the upstream of the flow element, and an exit section of $2 W_{p}$ was added to the downstream of the flow element. For the power generation from the solid fuel grain, a clipped cosine distribution in the radial direction (maximum power at the center of the innermost flow element, and zero power at the edge of the outermost flow element) was specified. Two distribution curves of power generation $(\phi)$ in the axial direction were investigated. Case \#1 has a pure sine distribution and Case \#2 has a clipped sine distribution, and both are shown in the following

$$
\begin{aligned}
& \text { Case \#1: } \quad \phi=\phi_{\max } \sin \left(\pi x / L_{p}\right) \quad x: 0 \rightarrow L_{p} \\
& \text { Case \#2: } \phi=\phi_{\max } \min \left[1,1.5 \sin \left(\pi x / L_{p}\right)\right] \quad x: 0 \rightarrow L_{p}
\end{aligned}
$$

where $\phi_{\max }$ is the maximum power and $L_{p}$ is the length of the flow element. The total power generated is set to be $491 \mathrm{~kW}$, which is about $80 \%$ of the designed value ${ }^{31}$. The reason for selecting this value is because this power is high enough to cause flow chocking in the flow element. Further increase of the power will lead to unrealistic numerical solutions because of the fixed mass inlet boundary condition and mass conservation exit boundary condition. This set of boundary conditions was used because only a $1 / 6$ segment of one flow element was simulated and thus the nozzle (which fits multiple flow elements) can not included. Since each flow element has different energy input, the centermost flow element, subjected to highest heat addition, is simulated in the present study as the worst scenario. Furthermore, the purpose of this study is to investigate possible occurrence of flow chocking within the flow channel, what is the maximum flow Mach number within the flow channel at the designed power level is a secondary issue and will be a subject for the future study. The heat transfer between the fluid flow and the solid fuel is simulated using the CHT model. A single elementary reaction was employed to model the hydrogen dissociation process due to the high temperature. 


\begin{tabular}{|c|c|c|c|c|c|}
\hline \multicolumn{3}{|c|}{ Flow Element Geometry } & \multicolumn{3}{c|}{ Inflow conditions $\left(\mathrm{H}_{2}\right)$} \\
\hline $\begin{array}{c}\text { Element width } \\
\left(W_{p}\right)\end{array}$ & $\begin{array}{c}\text { Channel } \\
\text { diameter }\left(D_{p}\right)\end{array}$ & $\begin{array}{c}\text { Element length } \\
\left(L_{p}\right)\end{array}$ & Pressure & Temperature & Mass flow rate \\
\hline $19.1 \mathrm{~mm}$ & $2.05 \mathrm{~mm}$ & $890 \mathrm{~mm}$ & $39.05 \mathrm{~atm}$ & $279 \mathrm{~K}$ & $0.0147 \mathrm{~kg} / \mathrm{s} / \mathrm{element}$ \\
\hline
\end{tabular}

Table 1. Geometry of a flow element and coolant flow inlet conditions

\begin{tabular}{|l|c|c|c|}
\hline & Thermal conductivity & Density & Specific heat \\
\hline UC-C-ZrC composite & $91 \mathrm{~W} / \mathrm{m} \cdot \mathrm{K}$ & $6142 \mathrm{~kg} / \mathrm{m}^{3}$ & $468.4 \mathrm{~W} \cdot \mathrm{s} / \mathrm{kg} \cdot \mathrm{K}$ \\
\hline $\mathrm{ZrC}$ composite & $19 \mathrm{~W} / \mathrm{m} \cdot \mathrm{K}$ & $6531 \mathrm{~kg} / \mathrm{m}^{3}$ & $368 \mathrm{~W} \cdot \mathrm{s} / \mathrm{kg} \cdot \mathrm{K}$ \\
\hline
\end{tabular}

Table 2. Thermal properties of flow element and coating layer at $300 \mathrm{~K}$

The numerical results of power generation cases \#1 and \#2 are plotted as shown in Figures 13-18. Figure 13 shows the pressure, temperature and Mach number contours at the symmetry boundaries and solid-fluid interfaces of the power generation case \#1, while Figure 16 shows those features of the power generation case \#2, respectively. As can be seen from the Mach number contours, the coolant flow is choked near the end of the flow channel. It is noted that these two plots are re-scaled due to extremely large aspect (length-to-width) ratios such that the contours are viewable and distinguishable. Figure 14 shows the pressure distributions along the centerlines of the center and outer flow channels, and temperature distributions along 7 axial lines of the power generation case \#1. The location of each line is depicted in Figure 5 (Line \#1: centerlines of the center flow channel; Line \#2: the line along the midpoint of the coating layer around the center flow channel; Line \#3: the line along the mid-point between the center and outer flow channels; Line \#4: the line along the inner mid-point of the coating layer around the outer flow channel; Line \#5: centerlines of the outer flow channel; Line \#6: the line along the outer mid-point of the coating layer around the outer flow channel; Line \#7: the line between the outer flow channel and the edge of the flow element). In the temperature distribution plot, the location of the maximum temperature gradient along each line is also marked with a symbol which has the same color as each line. It can be seen that Lines \#2 and \#4 have the same maximum temperature gradient locations, which are very close to that of Line \#3. The axial temperature distribution is qualitatively similar to that of 1-D calculation. Some researchers think that the location of maximum temperature gradient is critically linked to the potential corrosion of the flow element caused by different thermal expansion coefficients between the coating layer and the fuel. Hence, temperature distributions in the radial direction (across the solid-fluid interface) at two maximum temperature gradient locations (of the coating layer and center flow channel) are also plotted as shown in Figure 15. These two axial locations can be seen in Figure 14. The radial temperature profiles reveal that substantial thermal gradients occur at the fluid-coating interface and the coating-fuel interface. This may be attributed to large difference of the thermal conductivities between the coating material and the fuel, as shown in Table 2. Though 1-D calculations are efficient and can provide estimation of axial temperature distributions and maximum thermal gradient locations, whether the mid-section corrosion will occur can only be speculated because no information about the temperature gradients across different materials and fluid is available for thermal stress and fracture analyses. This shows the need for conducting 3-D CFD simulations in order to obtain a detailed thermal-fluid environment in the flow element. It is noted that this is a steady-state result, and thus the thermal gradient could be much stronger during transient startup as pointed out by Wang et al. ${ }^{9}$ Figure 17 shows axial temperature distributions of the power generation case \#2, which are similar to those of Figure 14 except that the locations of maximum thermal gradient are further upstream than those of case \#1. This is expected because power generation case $\# 2$ has a clipped sine distribution, where the power rises up to its peak value faster in the axial direction. Temperature distributions in the radial direction at the same two maximum thermal gradient locations are plotted in Figure 18. Substantial thermal gradients at the fluid-coating interface and the coating-fuel interface also can be observed. However, the level of thermal gradient at the coating-fuel interface is relatively smaller than that of the power generation case \#1. This can be attributed to the characteristics of the clipped cosine distribution, which has a larger area subjected to the peak power source but has a smaller peak power (such that the overall energy inputs for both cases are the same). The exit temperatures of both cases are almost identical, and have a value about $2660 \mathrm{~K}$. 


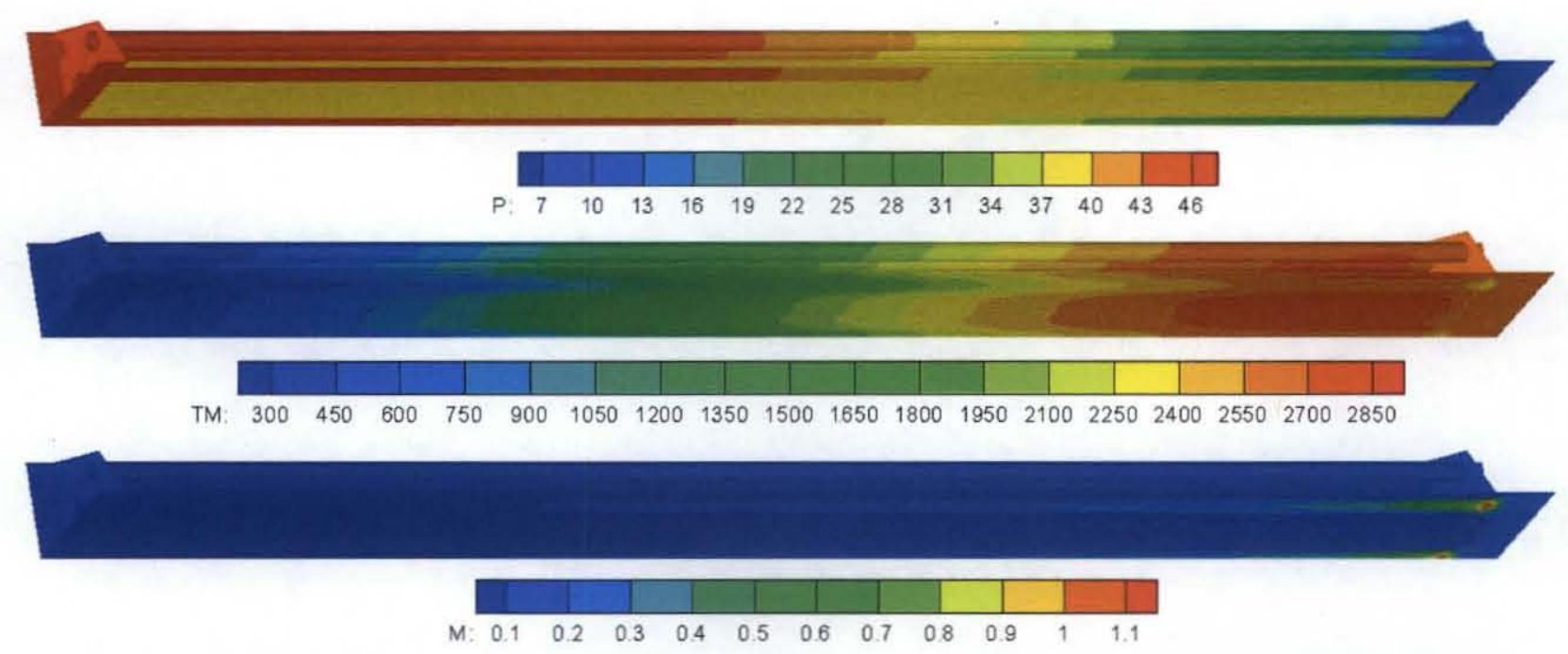

Figure 13. Pressure (top, in atm), temperature (middle, in K), and Mach number (bottom) contours at the boundary and solid-fluid interfaces of power generation case \#1
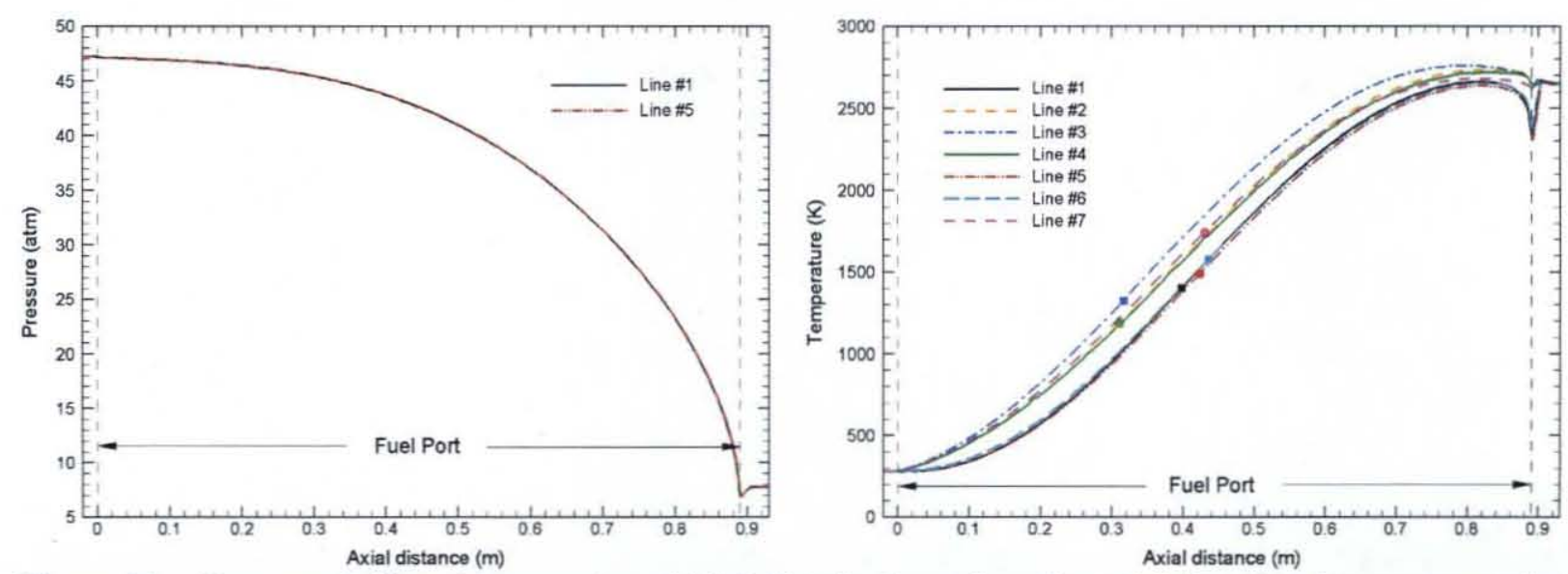

Figure 14. Pressure (left) and temperature (right) distributions along the axial direction (power generation case \#1)
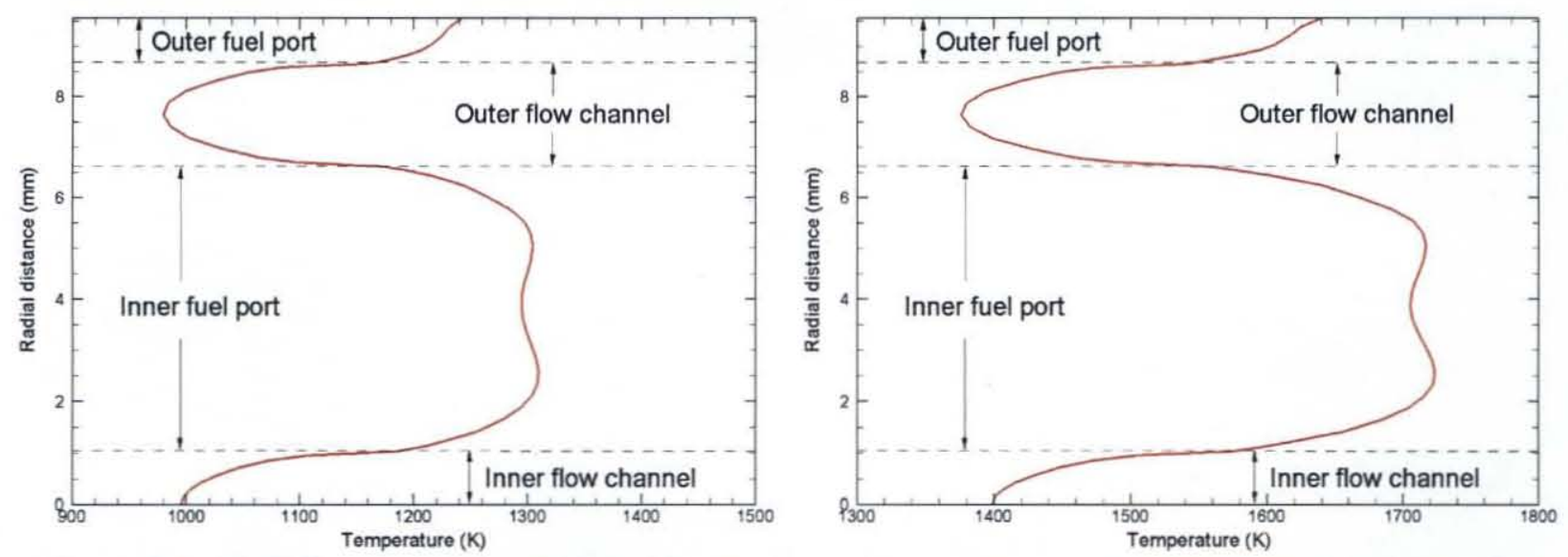

Figure 15. Radial temperature profiles at the maximum temperature gradient locations of coating layer (left) and center flow channel (right) for power generation case \#1 

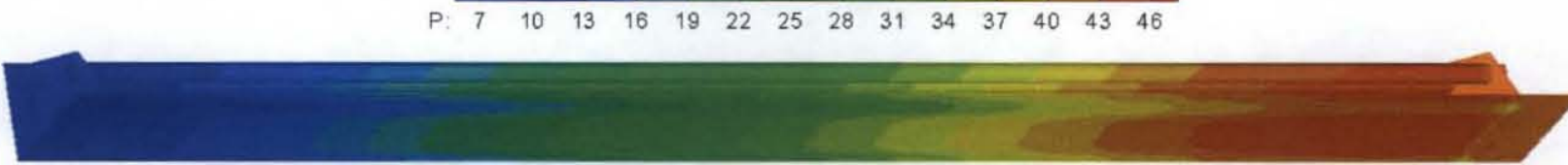

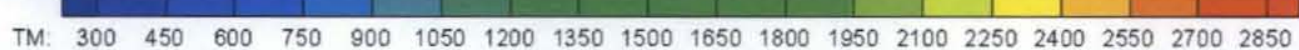

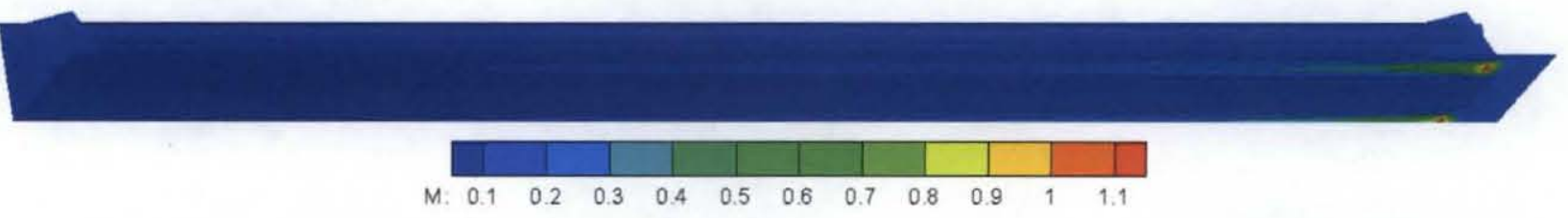

Figure 16. Pressure (top, in atm), temperature (middle, in $\mathrm{K}$ ), and Mach number (bottom) contours at the boundary and solid-fluid interfaces of power generation case \#2
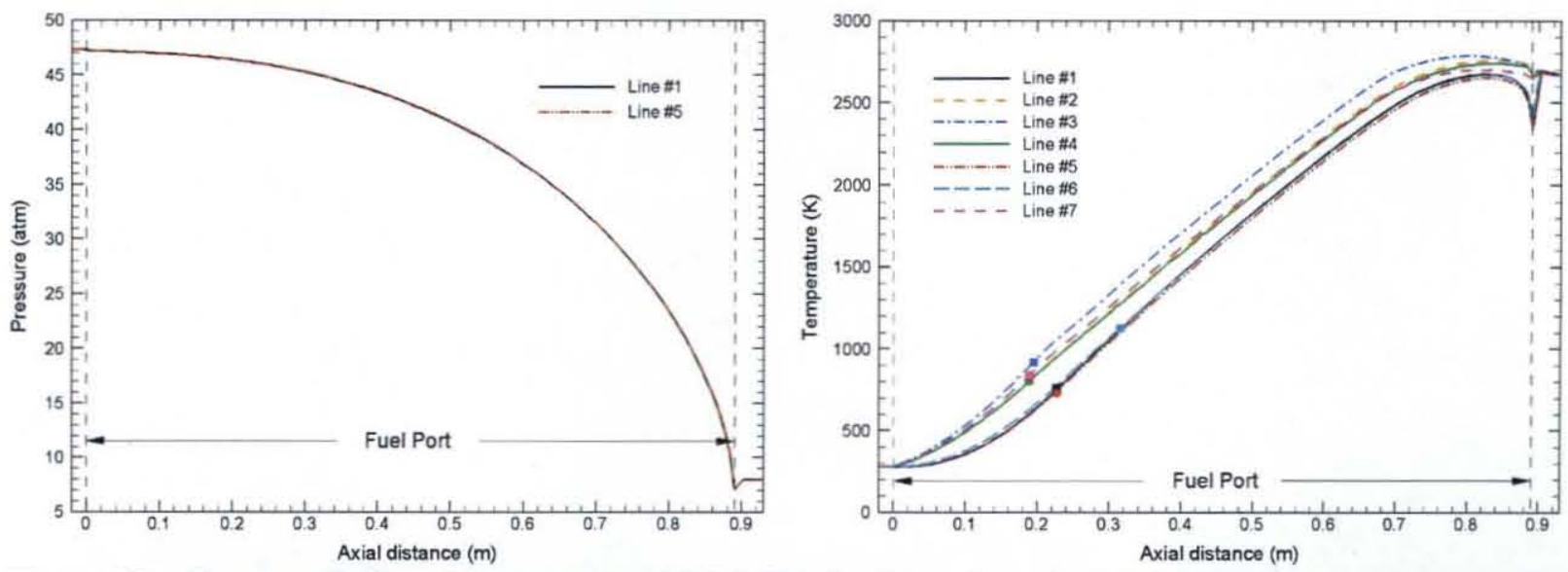

Figure 17. Pressure (left) and temperature (right) distributions along the axial direction (power generation case \#2)
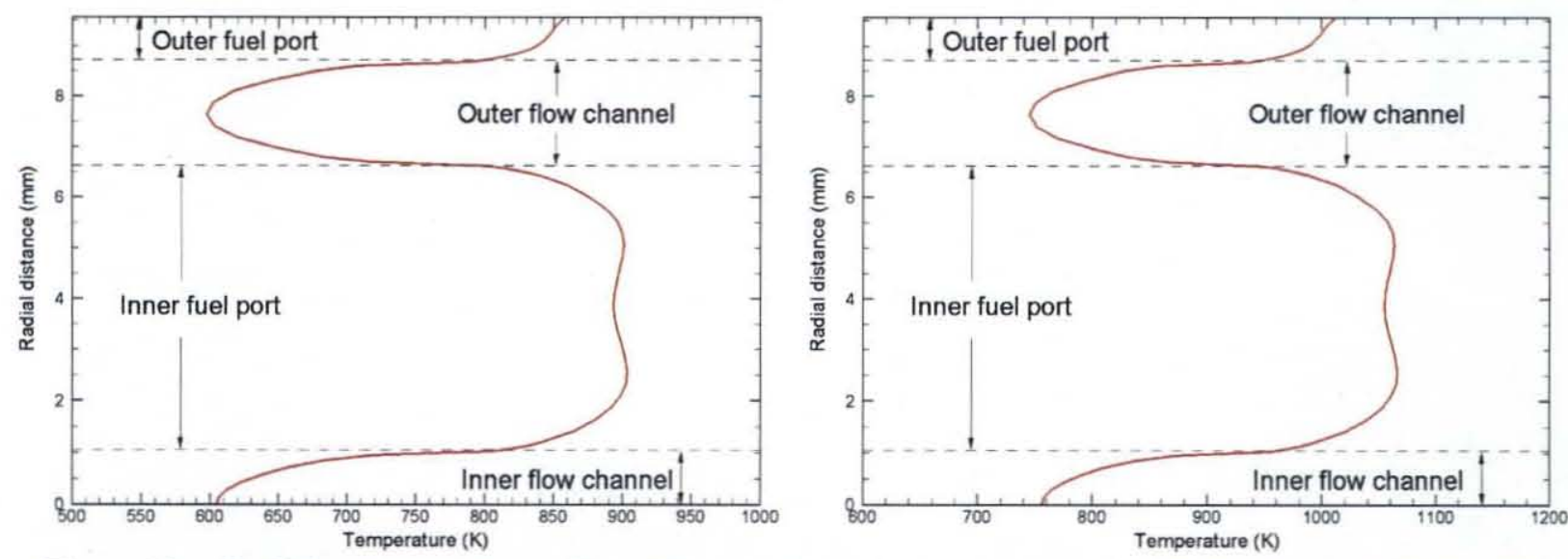

Figure 18. Radial temperature profiles at the maximum temperature gradient locations of coating layer (left) and center flow channel (right) for power generation case \#2 


\section{Conclusions}

In the present study, the developed CFD model (including conjugate heat transfer, power generation, and porosity model) has been successfully applied to simulate the thermal-fluid environment of a single flow element in a nuclear thermal thrust chamber. The use of the porosity model shows a promise of being an efficient numerical approach for simulating an assembly of a huge number of flow elements. However, the employed porosity model fails to match the numerical data of the $\mathrm{CHT}$ model at a wide range of operating and geometric conditions. Further study is required to improve the porosity model such that it can be employed to accurately simulate the global thermal-fluid environment of the flow element. A detailed numerical analysis of a powered flow element using the CHT model provides the insight of the thermal-fluid environment. The numerical result indicates large thermal gradients at the fluid-coating and coating-fuel interfaces for the selected fuel and coating materials, which can potentially cause the mid-section corrosion problem. Moreover, the radial temperature profiles show that 3-D CFD simulations provide critical information which can not be obtained from 1-D calculations. Selection of different material may be able to reduce the level of thermal gradients. The results also reveal that the power distribution function can affect the location and level of maximum thermal gradient. Nevertheless, the numerical results of both power distributions indicate that even at $80 \%$ of the designed power level some flow elements possibly have flow chocking in the flow channel and some may not depending on the local heat source magnitude. The occurrence of flow chocking in some flow channels can cause non-uniform flow distributions among flow elements, and produce undesirable side load. Further study using 3-D CFD simulations of the full configuration of the flow element assembly with a downstream nozzle is needed in order to evaluate 1) the magnitudes of the maximum flow Mach number, 2) distributions of mass flow rates pressure drops among the flow elements, and 3) the magnitude of side load and its impact. Different flow element configurations should also be examined in the future to eliminate the flow chocking problem.

\section{Acknowledgments}

This study was supported by a Nuclear Systems Office task entitled "Multiphysics Thrust Chamber Modeling" of which Wayne Bordelon was the project manager. Steve Simpson and Karl Nelson provided operating conditions for the hypothetical nuclear thermal engine. Bill Emrich suggested the cosine power profile. Thermal properties provided by Panda Binayak, Robert Hickman, and Bill Emrich are also acknowledged.

\section{References}

${ }^{1}$ Koenig, D.R., "Experience Gained from the Space Nuclear Rocket Program (Rover)," LA-10062-H, Los Alamos National Laboratory, Los Alamos, New Mexico, 1986.

${ }^{2}$ Lyon, L.L., "Performance of (U, Zr)C-Graphite (Composite) and of $(\mathrm{U}, \mathrm{Zr}) \mathrm{C}$ (Carbide) Fuel Elements in the Nuclear Furnace 1 Test Reactor," LA-5398-MS, Los Alamos Scientific Laboratory, Los Alamos, New Mexico, 1973.

${ }^{3}$ Taub, J.M., "A Review of Fuel Element Development for Nuclear Rocket Engine," Los Alamos Report LA5931, June 1975.

${ }^{4}$ Chen, Y.-S., Liu, J., Zhang, S., and Mallapragada, P., "An Integrated Tool for Launch Vehicle Base-Heating Analysis," Final Report, NAS8-00002, Engineering Sciences, Inc., Huntsville, AL, 2001.

${ }^{5}$ Chen, Y.S., "An Unstructured Finite Volume Method for Viscous Flow Computations", $7^{\text {th }}$ International Conference on Finite Element Methods in Flow Problems, University of Alabama in Huntsville, Huntsville, Alabama, Feb. 3-7, 1989.

${ }^{6}$ Shang, H.M., Shih, M.H., Chen, Y.S., and Liaw, P., "Flow Calculation on Unstructured Grids with a PressureBased Method," Proceedings of $6^{\text {th }}$ International Symposium on Computational Fluid Dynamics, Lake Tahoe, NV, Sep. 4-8, 1995.

${ }^{7}$ Chen. Y.-S., Zhang S., and Liu, J., "Stage Separation Performance Analysis Project," Final Report, H-34345D, Engineering Sciences, Inc., Huntsville, AL, 2002.

${ }^{8}$ Wang, T.S., Canabal, F., Cheng, G.C., and Chen, Y.S., "Multiphysics Analysis of a Solid-Core Nuclear Thermal Engine Thrust Chamber," AIAA Paper 2006-2927, $9^{\text {th }}$ AIAA/ASME Joint Thermophysics and Heat Transfer Conference, June 5-8, 2006.

${ }^{9}$ Wang, T.S., Luong, V., Foote, J., and Chen, Y.S., "Analysis of a Cylindrical Specimen Heated by an Impinging Hot Hydrogen Jet," AIAA Paper 2006-2926, $9^{\text {th }}$ AIAA/ASME Joint Thermophysics and Heat Transfer Conference, June 5-8, 2006. 
${ }^{10}$ Gaski, J., "The Systems Improved Numerical Differencing Analyzer (SINDA) Code - a User's Manual," Aerospace Corp., El Segundo, CA, Feb. 1986

"Launder, B.E. and Spalding, D.B., "The Numerical Calculation of Turbulent Flows," Computer Methods in Applied Mechanics and Engineering, Vol. 3(2), 1974, pp. 269-289.

${ }^{12}$ Chen, Y.-S., and Kim, S. W., "Computation of Turbulent Flows Using an Extended k- $\varepsilon$ Turbulence Closure Model," NASA CR-179204, 1987.

${ }^{13}$ Liakopoulos, A., "Explicit Representations of the Complete Velocity Profile in a Turbulent Boundary Layer," AIAA Journal, Vol. 22, No. 6, 1984, pp. 844-846.

${ }^{14}$ Viegas, J.R., Rubesin, M.W., and Horstman, C.C., "On the Use of Wall Function as Boundary Conditions for Two-Dimensional Separated Compressible Flows," AIAA Paper 85-0180, AIAA $23^{\text {rd }}$ Aerospace Sciences Meeting, Jan. 14-17, 1985.

${ }^{15}$ Karki, K.C., and Patankar, S.V., "Pressure Based Calculation Procedure for Viscous Flows at all Speeds in Arbitrary Configurations," AIAA J., Vol. 27, 1989, pp. 1167-1174.

${ }^{16}$ Patankar, S.V., Numerical Heat Transfer and Fluid Flow, Hemisphere, New York, 1980.

${ }^{17}$ Barth, T.J., and Jespersen, D.C., "The Design and Application of Upwind Schemes on Unstructured Meshes," AIAA Paper 89-0366, 1989.

${ }^{18}$ Rhie, C.M. and Chow, W.L., "A Numerical Study of the Turbulent Flow past an Isolated Airfoil with Trailing Edge Separation," AIAA Journal, Vol. 21, 1983, pp. 1525-1532.

${ }^{19}$ Van Der Vorst, H.A., "Bi-CGSTAB: A Fast and Smoothly Converging Variant of Bi-CG for the Solution of Nonsymmetric Linear Systems,"SIAM J. Sci. Stat. Comput., Vol. 13(2), 1992, pp. 631-644.

${ }^{20}$ Saad,Y. and Schultz, M.H., "GMRES: A Generalized Minimal Residual Algorithm for Solving Nonsymmetric Linear Systems," SIAM J. Sci. Stat. Comput., Vol. 7(3), 1986, pp. 856-869.

${ }^{21}$ Raw, M., "Robustness of Coupled Algebric Multigrid for the Navier-Stkoes Equations," AIAA Paper 96-0297, 1996.

${ }^{22}$ W. Gropp, E. Lusk, and A. Skjellum, "Using MPP”, published by MIT Press, ISBN 0-262-57104-8.

${ }^{23}$ Karypis and V. Kumar, "METIS, A Software Package for Partitioning Unstructured Graphs, Partitioning Meshes, and Computing Fill-Reducing Orderings of Sparse Matrices," Version 3.0.3, November 5, 1997.

${ }^{24}$ Bird, R.B., Stewart, W.E., and Lightfoot, E.N., "Transport Phenomena," $2^{\text {nd }}$ Ed., John Wiley \& Sons, Inc.

${ }^{25}$ Ross, D.H., Dillavou, M., Gopalsamy, S., Shum, P.C., and Shih, A.M., "A Framework for Developing Geometry-Grid Templates for Propulsion Elements," $16^{\text {th }}$ NASA Thermal and Fluids Analysis Workshop, Orlando, FL, August 8-12, 2005.

${ }^{26}$ Shih, A. M., Gopalsamy, S., Ito, Y., Ross, D. H., Dillavou, M. and Soni, B. K., "Automatic and Parametric Mesh Generation Approach," $17^{\text {th }}$ International Association for Mathematics and Computers in Simulation (IMACS) World Congress, Paris, France, July 2005.

${ }^{27}$ Shih, A.M., Ross, D.H., Dillavou, M., Gopalsamy, S., Soni, B.K., Peugeot, J.W., and Griffin, L.W., "A Geometry-Grid Generation Template Framework for Propellant Delivery System," $42^{\text {nd }}$ AIAA/ASME/SAE/ASEE Joint Propulsion Conference and Exhibit, Sacramento, CA, July, 2006.

${ }^{28}$ Ito, Y. and Nakahashi, K., "Direct Surface Triangulation Using Stereolithography Data," AIAA Journal, Vol. 40, No. 3, 2002, pp. 490-496.

${ }^{29}$ Ito, Y. and Nakahashi, K., "Surface Triangulation for Polygonal Models Based on CAD Data," International Journal for Numerical Methods in Fluids, Vol. 39, Issue 1, 2002, pp. 75-96.

${ }^{30}$ Ito, Y., Shih, A. M., Soni, B. K. and Nakahashi, K., "Multiple Marching Direction Approach to Generate High Quality Hybrid Meshes," AIAA Journal, Vol. 45, No. 1, 2007, pp. 162-167.

${ }^{31}$ Durham, F.P., "Nuclear Engine Definition Study Preliminary Report," LA-5044-MS, Los Alamos Scientific Laboratory, Los Alamos, New Mexico, 1972. 


\title{
Numerical Simulations of Single Flow Element in a Nuclear Thermal Thrust Chamber
}

\author{
AIAA 2007-4143
}

Gary C. Cheng, Yasushi Ito, Doug Ross

Mechanical Eng. Dept., UAB

Yen-Sen Chen

Engineering Sciences, Inc.

Ten-See Wang

NASA MSFC

$3^{\text {th }}$ AIAA Thermophysics Conference

June 25-28, 2007, Miami, Florida 


\section{Acknowledgment}

- Supported by NASA Nuclear Systems Office task entitled "Multiphysics Chamber Modeling"

- Project manager: Wayne Bordelon

- Operating conditions provided by Steve Simpson \& Carl Nelson

- Thermal properties provided by Panda Binayak, Robert Hickman, and Bill Emrich

- Power generation profile suggested by Bill Emrich 


\section{BACKGROUND}

- Nuclear thermal rocket is one of the candidate propulsion system for future space exploration

- Study from Rover/NERVA program showed the solid-core reactor is a feasible concept to produce $I_{\text {sp }}$ over $850 \mathrm{sec}$

- The solid-core reactor consists of hundreds of heat generating solid flow elements

- Each flow element contains tens of flow channels through which $\mathrm{H}_{2}$ is heated up before entering a nozzle

- To achieve maximum efficiency:

- The reactor operates at very high temperature and power density

- Number and area of flow channels per flow element need to be optimized 


\section{BACKGROUND (Cont.)}

- A coating layer was designed to protect the solid fuel from chemical attack by hot $\mathrm{H}_{2}$

- Results of Rover/NERVA tests indicate:

-Mid -section corrosion (crack in coating layer) occurs if the solid fuel temperature exceeds certain value, which leads to mass loss of the flow element

-Mid -section corrosion was suspected to be caused by different thermal expansions between the flow element and coating layer

-Dispa rity of thermal expansion can be large if large thermal gradients occur near the interface between the flow channel, coating layer and flow element

-Possible flow choking in some of the flow channels, which will lead to non-uniform propellant flow distributions among flow elements and undesirable side load 


\section{MOTIVATIONS}

- Performance of the solid-core reactor was previously estimated based on 1-D analysis, which lacks of detailed thermal-fluid environments to address the aforementioned problems

- 3-D CFD simulations can provide high-fidelity thermalfluid environments in the reactor, but need to

-Imp rove computational efficiency

-Couple calculation of heat transfer in the fluid flow with calculation of heat conduction in the solid fuel

- Includ e realistic power generation model

- Inco rporate correct thermal properties of the flow element and coating layer

- Accou $\mathrm{nt}$ for dissociation of $\mathrm{H}_{2}$ at high temperature

-Accou nt for neutronic reactions 


\section{OBJECTIVES}

- Develop an efficient, accurate CFD methodology to simulate thermal-fluid environments of a single flow element

- Improve the conjugate heat transfer model and implement the power generation model to accurately predict detailed thermal-fluid environment within flow element

- Temperature, pressure, velocity distributions and possible choking of coolant flow

- Thermal gradient in the solid and at the solid-fluid interface for possible corrosion/cracking

- Provide temperature distributions in the flow element for the thermal stress analysis

- Improve and tune the porosity to efficiently calculate global thermalfluid phenomena: overall pressure loss \& temperature rise of coolant flow 


\section{Numerical Approach \& Models}

- Unstructured-grid pressure-based method for solving NavierStokes or RANS equations

- Cell center, finite volume approach

- Compressible and incompressible flows with all speed regimes

- Barth-Jespersen's linear reconstruction approach

- Conjugate Gradient, or GMRES Matrix Solvers

- PISO scheme for unsteady flow simulations

- Algebraic multi-grid (AMG) method to enhance convergence

- Flexible mesh adaptation refinement

- Parallel computing with MPI or PVM

- Dynamic memory allocations

- Domain decomposition with METIS 


\section{Numerical Approach \& Models (Cont.)}

- Standard and extended $k-\varepsilon$ model for modeling turbulence effects

- Finite-Rate and Equilibrium Chemistry Models

- Conjugate heat transfer model for coupling heat convection and conduction

- Heterogeneous spray model with Eulerian/Lagrangian framework and volume of fluid approach

- Homogeneous spray model with Eulerian/Eulerian framework and real-fluid property model

- Finite volume method for solving radiation transport equation with either gray gas or narrow band model

- Porosity model for flow through porous media

- Account for thermal non-equilibrium effect by solving vibrational energy equation 


\section{Governing Equations}

$$
\begin{aligned}
& \frac{\partial \rho}{\partial t}+\frac{\partial}{\partial x_{j}}\left(\rho V_{j}\right)=0 \\
& \frac{\partial\left(\rho V_{i}\right)}{\partial t}+\frac{\partial}{\partial x_{j}}\left(\rho V_{j} V_{i}\right)=-\frac{\partial p}{\partial x_{i}}+\frac{\partial \tau_{j i}}{\partial x_{j}}+S_{V} \\
& \frac{\partial\left(\rho h_{i}\right)}{\partial t}+\frac{\partial}{\partial x_{j}}\left(\rho V_{j} h_{t}\right)=\frac{\partial p}{\partial t}+\frac{\partial}{\partial x_{j}}\left[\left(\frac{\mu}{\operatorname{Pr}}+\frac{\mu_{t}}{\operatorname{Pr}_{i}}\right) \frac{\partial h}{\partial x_{j}}\right]+\frac{\partial \tau_{j i} V_{i}}{\partial x_{j}}+S_{h} \\
& \frac{\partial \rho C_{p} T}{\partial t}-\frac{\partial}{\partial x_{i}}\left(\kappa \frac{\partial T}{\partial x_{i}}\right)=Q_{v}+\frac{\partial Q_{i}}{\partial x_{i}} \\
& \frac{\partial\left(\rho \alpha_{i}\right)}{\partial t}+\frac{\partial}{\partial x_{j}}\left(\rho V_{j} \alpha_{i}\right)=\frac{\partial}{\partial x_{j}}\left[\left(\frac{\mu}{S c}+\frac{\mu_{t}}{S c_{i}}\right) \frac{\partial \alpha_{i}}{\partial x_{j}}\right]+S_{i}
\end{aligned}
$$




\section{Conjugate Heat Transfer}

$$
\begin{aligned}
& \frac{\partial\left(\rho h_{t}\right)}{\partial t}+\frac{\partial}{\partial x_{j}}\left(\rho V_{j} h_{t}\right)=\frac{\partial p}{\partial t}+\frac{\partial}{\partial x_{j}}\left[\left(\frac{\mu}{\operatorname{Pr}}+\frac{\mu_{t}}{\operatorname{Pr}_{i}}\right) \frac{\partial h}{\partial x_{j}}\right]+\frac{\partial \tau_{j i} V_{i}}{\partial x_{j}}+S_{h} \\
& \frac{\partial \rho C_{p} T}{\partial t}-\frac{\partial}{\partial x_{i}}\left(\kappa \frac{\partial T}{\partial x_{i}}\right)=Q_{v}+\frac{\partial Q_{s}}{\partial x_{i}}
\end{aligned}
$$

- Implicit scheme:

$$
\frac{1}{2} \rho C_{p} \frac{T_{b}^{n+1}-T_{b}^{n}}{\Delta t}=\frac{1}{y_{n}}\left(k \frac{T_{c}^{n}-T_{h}^{n}}{y_{n}}-q_{w}\right)
$$

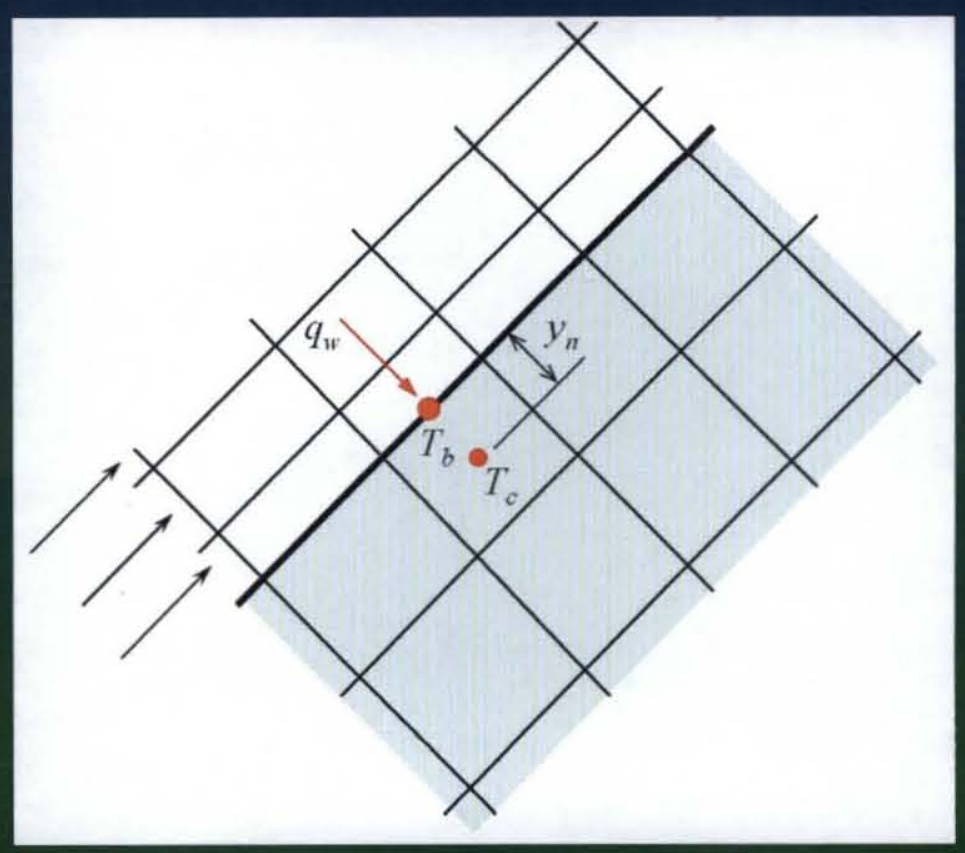




\section{Porosity Model}

$$
\begin{aligned}
& \frac{\partial\left(\rho V_{i}\right)}{\partial t}+\frac{\partial}{\partial x_{j}}\left(\rho V_{j} V_{i}\right)=-\frac{\partial p}{\partial x_{i}}+\frac{\partial \tau_{j i}}{\partial x_{j}}+S_{V} \\
& \vec{S}_{V}=\frac{D}{\xi \forall}=\frac{1}{2} \frac{\rho\left|V_{s}\right| C_{D}}{\xi^{2} d / 4} \vec{V}_{s} \text { and } C_{D}=f_{D} C_{f} \\
& C_{f}=0.0791 \operatorname{Re}_{d}^{-0.25} \quad \text { where } \quad \operatorname{Re}_{d}=\frac{\rho V_{s} d}{\mu \xi} \\
& \frac{\partial\left(\rho h_{t}\right)}{\partial t}+\frac{\partial}{\partial x_{j}}\left(\rho V_{j} h_{l}\right)=\frac{\partial p}{\partial t}+\frac{\partial}{\partial x_{j}}\left[\left(\frac{\mu}{\operatorname{Pr}}+\frac{\mu_{t}}{\operatorname{Pr}_{i}}\right) \frac{\partial h}{\partial x_{j}}\right]+\frac{\partial \tau_{j i} V_{i}}{\partial x_{j}}+S_{h} \\
& \frac{\partial \rho C_{p} T}{\partial t}-\frac{\partial}{\partial x_{i}}\left(\kappa \frac{\partial T}{\partial x_{i}}\right)=Q_{v}+\frac{\partial Q_{s}}{\partial x_{i}} \\
& S_{h}=\frac{Q}{\xi \forall}=\frac{f_{h}}{2} \frac{\rho\left|V_{s}\right| C_{f}}{\operatorname{Pr}^{2 / 3} \xi d / 4} C_{p} \Delta T \quad \text { where } \quad \Delta T=T_{s}-T_{g} \\
& Q_{v}=\frac{Q+Q_{p}}{(1-\xi) \forall}=\frac{f_{h h}}{2} \frac{\rho|V| C_{f}}{\operatorname{Pr}^{2 / 3}(1-\xi) d / 4} C_{p} \Delta T+\frac{q_{p}}{(1-\xi)} \quad \text { and } \quad \Delta T=T_{g}-T_{s} ; \quad q_{p}=\frac{Q_{p}}{\forall}
\end{aligned}
$$




\section{Geometry of Flow Element Assembly}

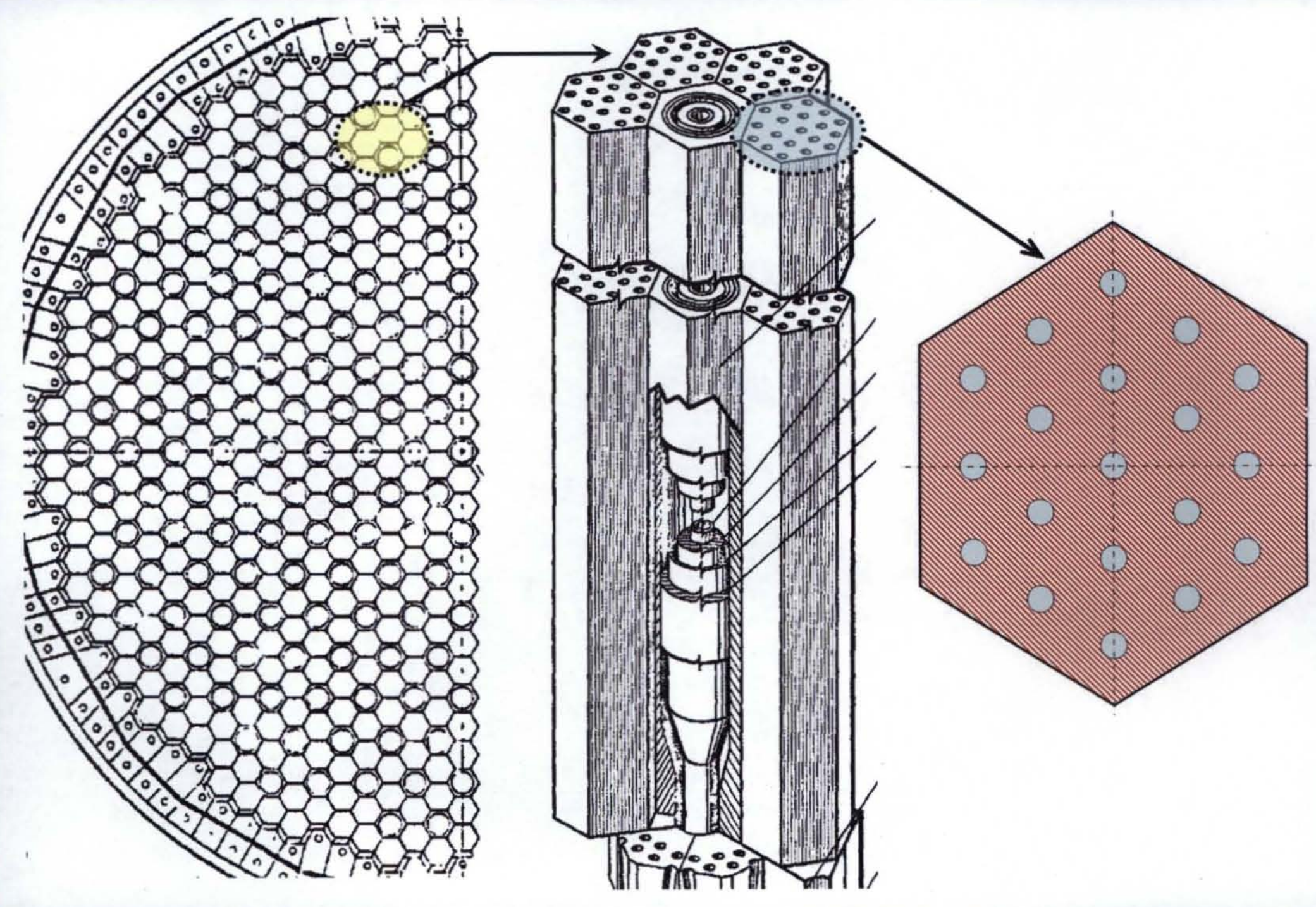


Mesh of Single Flow Element
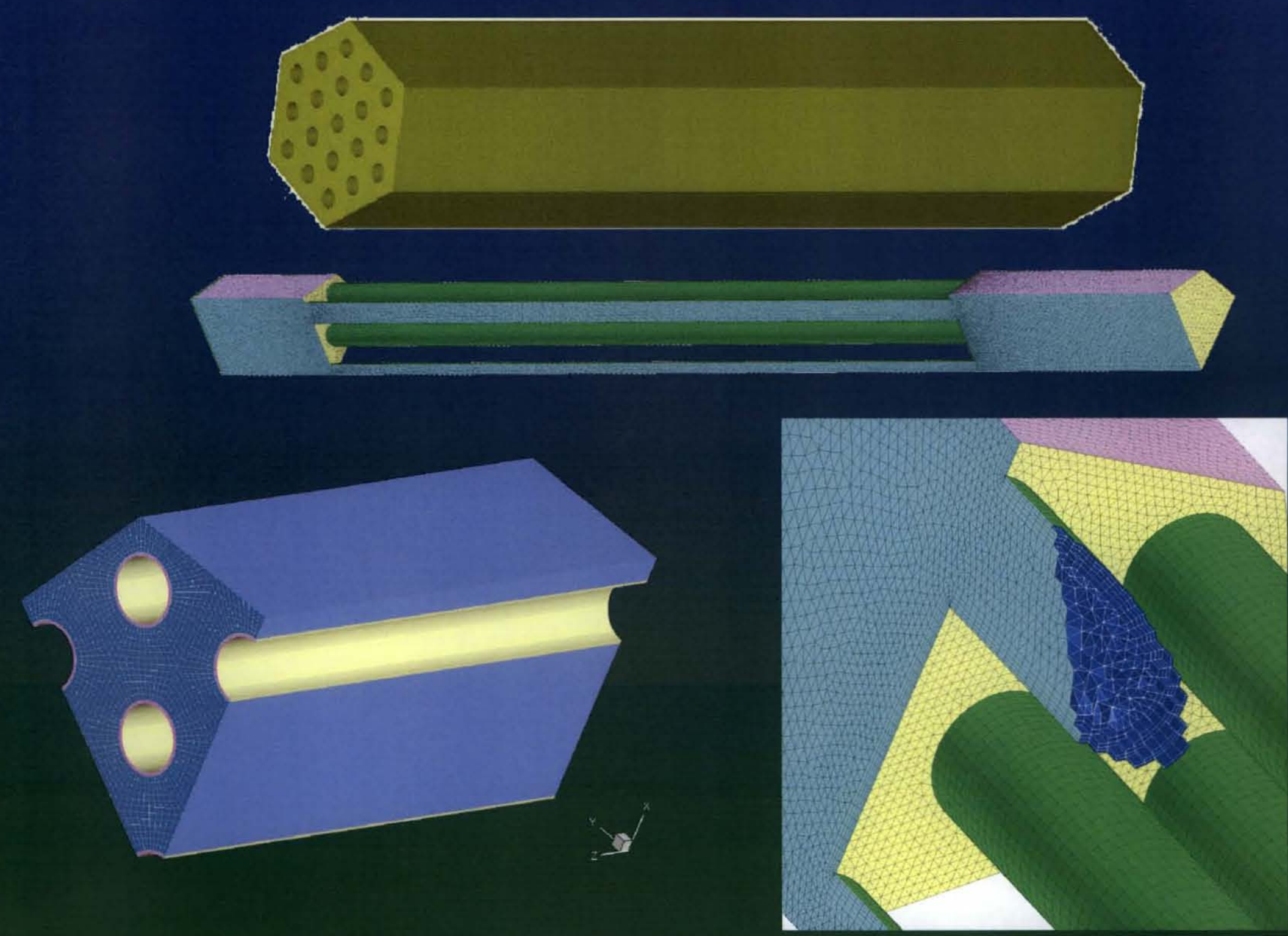


\section{Tuning of Porosity Model}
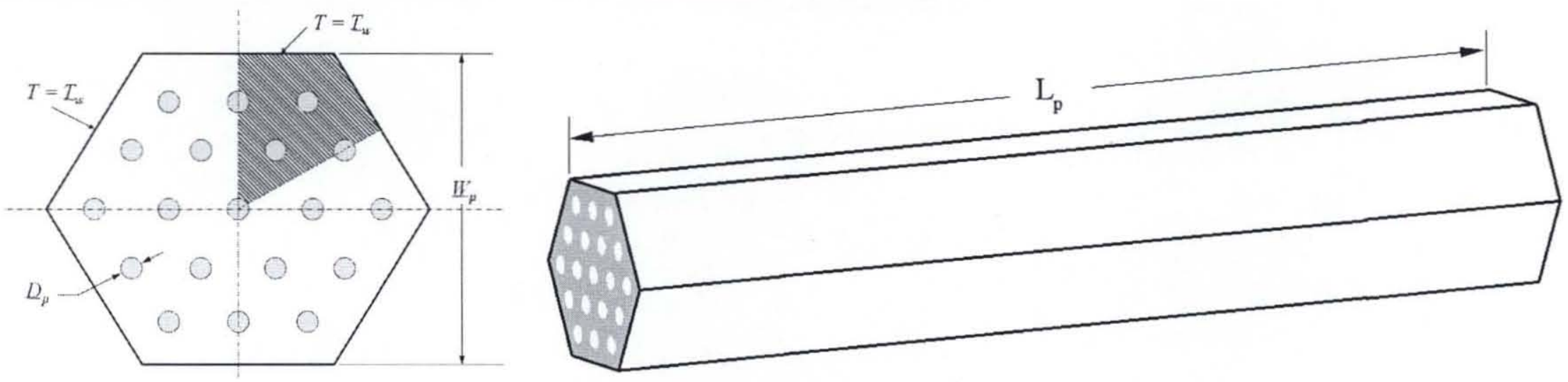

- $T_{w}: 1000,2000$, and $3000 \mathrm{~K}$

- $D_{p}: 2.05,2.29$, and $2.54 \mathrm{~mm}$

- $L_{p}: 110 \mathrm{~mm}, W_{p}: 19.1 \mathrm{~mm}$

- Flow element: (UC-C-ZrC composite) $\kappa=22.5 \mathrm{~W} / \mathrm{m} \cdot \mathrm{K}, \rho=13500 \mathrm{~kg} / \mathrm{m}^{3}, C_{p}=146.5 \mathrm{~W} \cdot \mathrm{s} / \mathrm{kg} \cdot \mathrm{K}$

- Inlet conditions:

Pressure: 34 atm, Temperature: 279 K, Flow rate: 0.0147 kg/s 


\section{Tuning of Porosity Model}

Simulation with conjugate heat transfer model

No. of cells: $1.7 \mathrm{M}$, no. of grid points: $555 \mathrm{~K}$
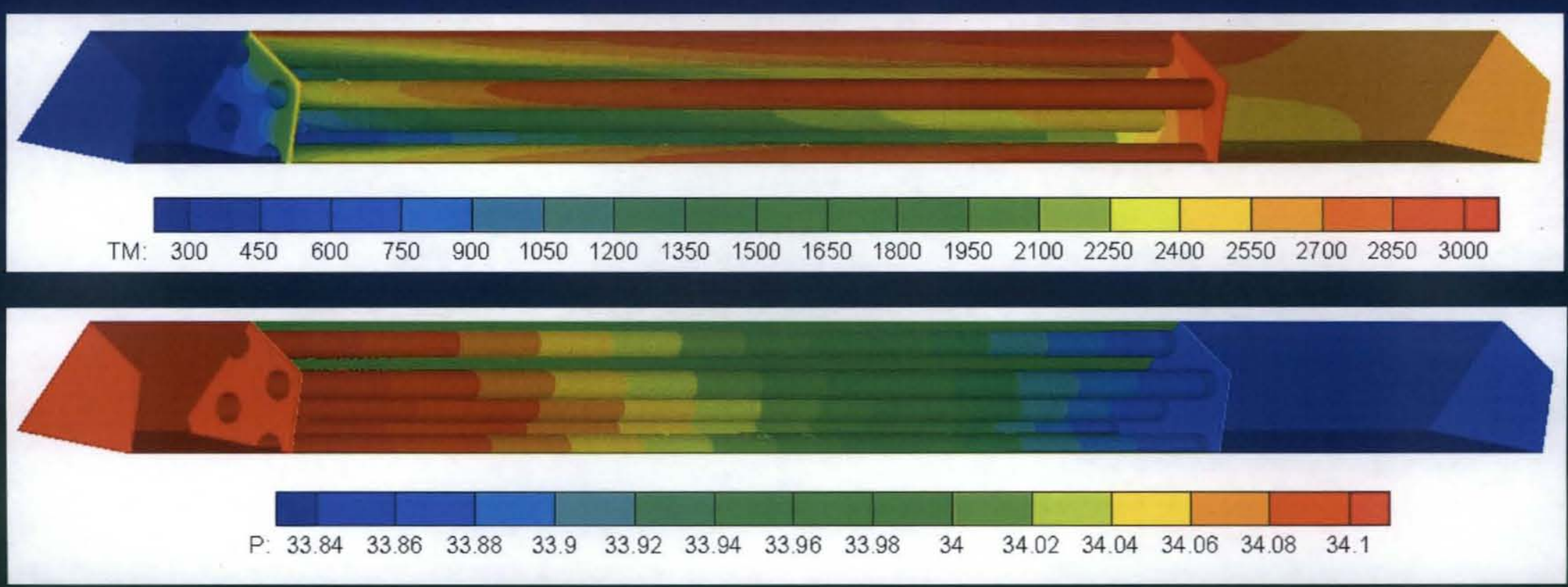
Tuning of Porosity Model
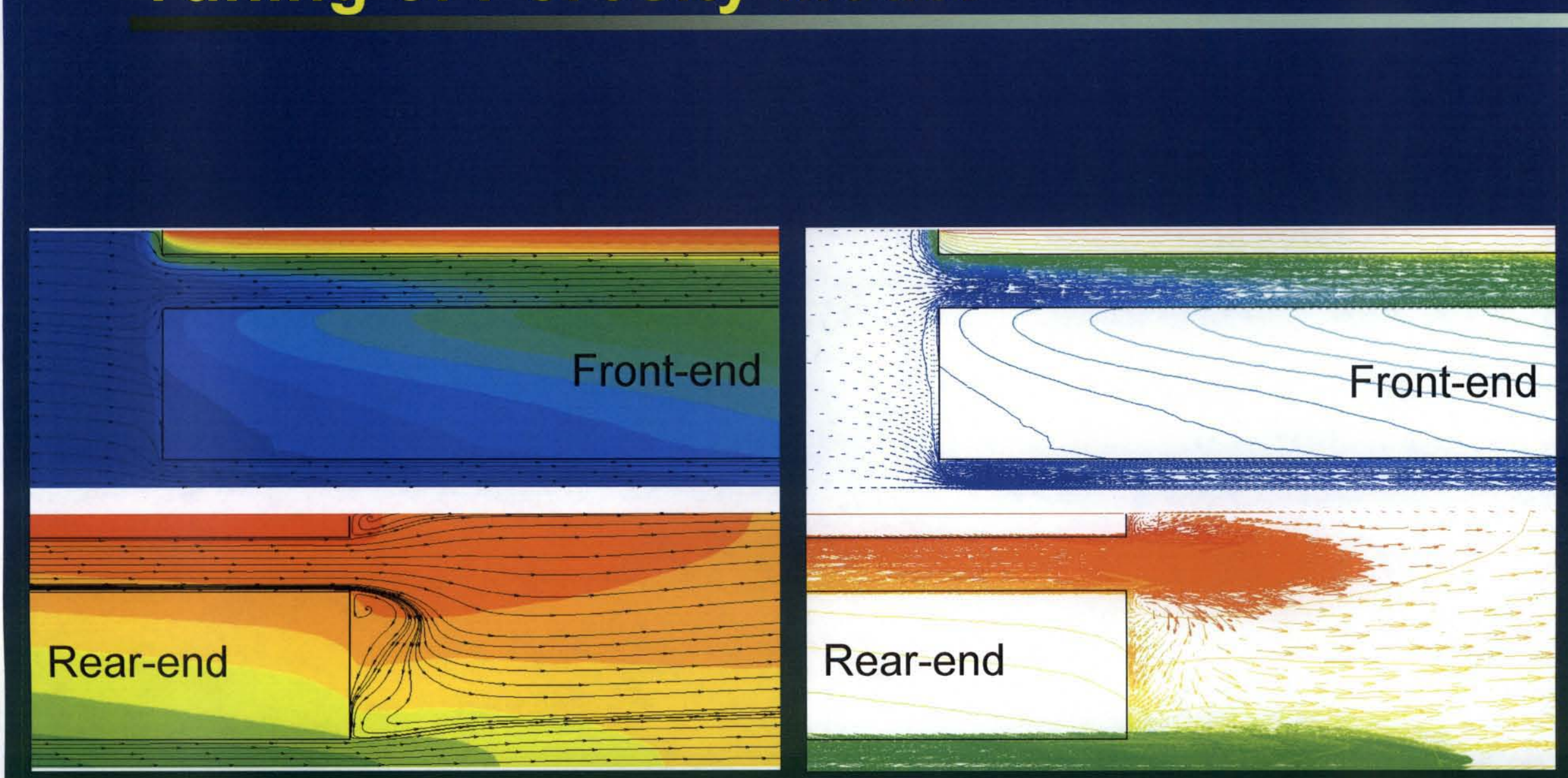


\section{Tuning of Porosity Model}
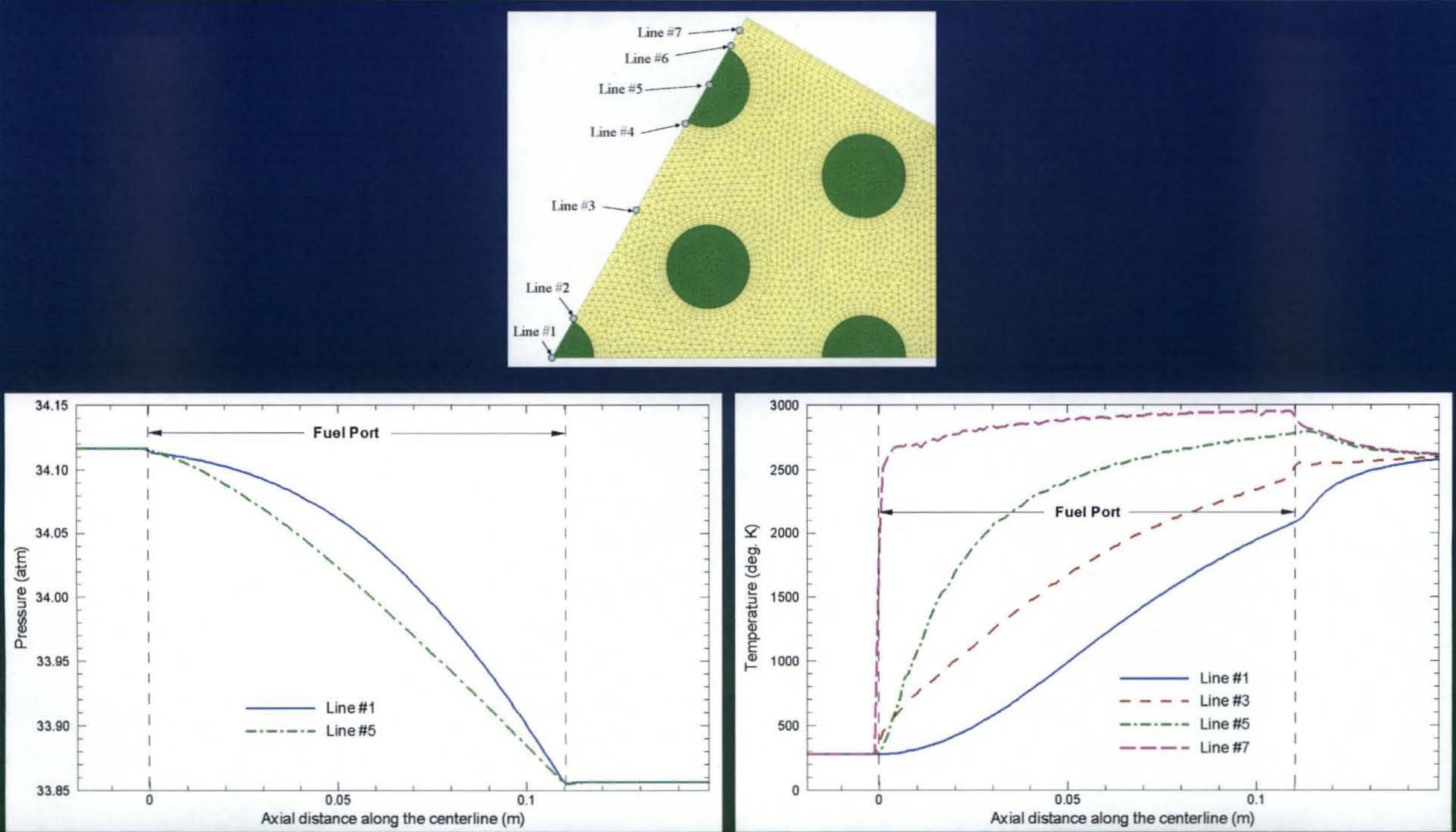


\section{Tuning of Porosity Model}

Simulation with porosity model

No. of cells: $22 \mathrm{~K}$, no. of grid points: $8.6 \mathrm{~K}$

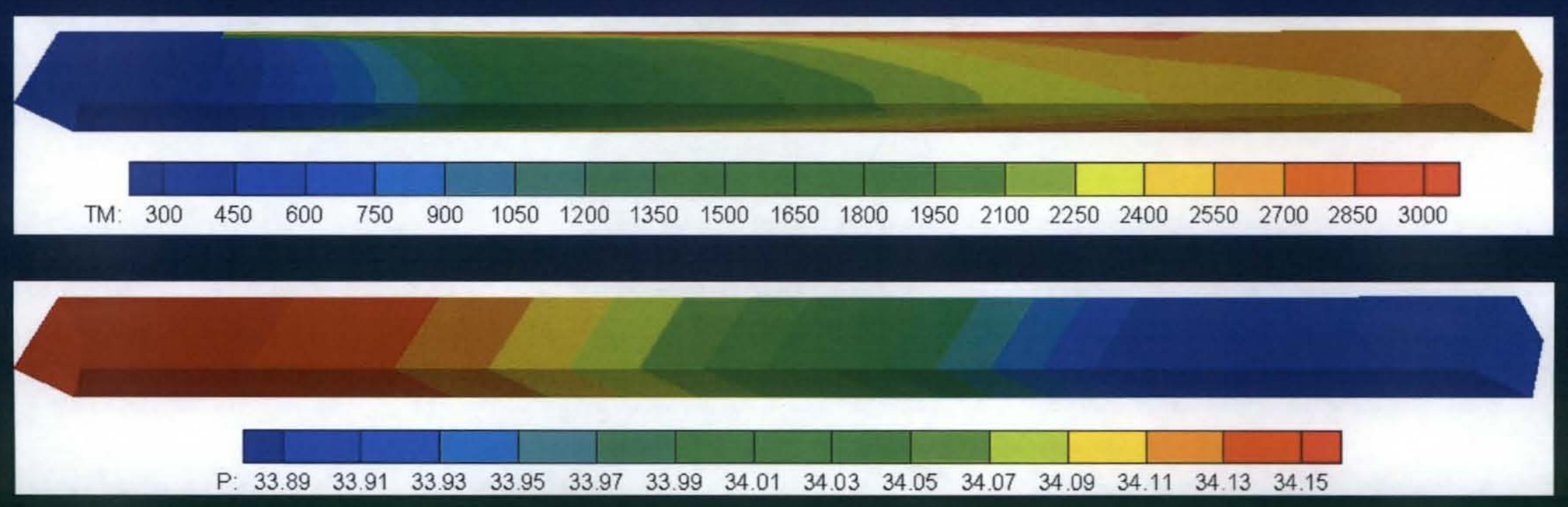




\section{Effect of Temperature}
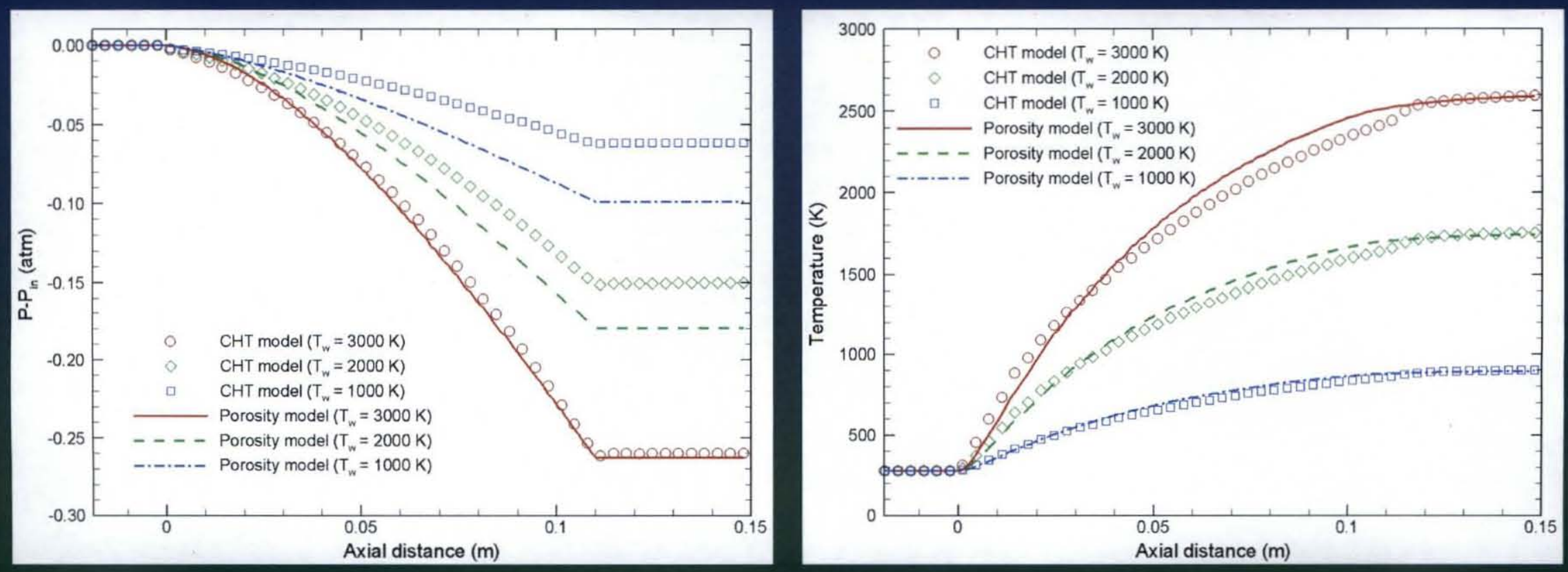


\section{Effect of Pore Size}
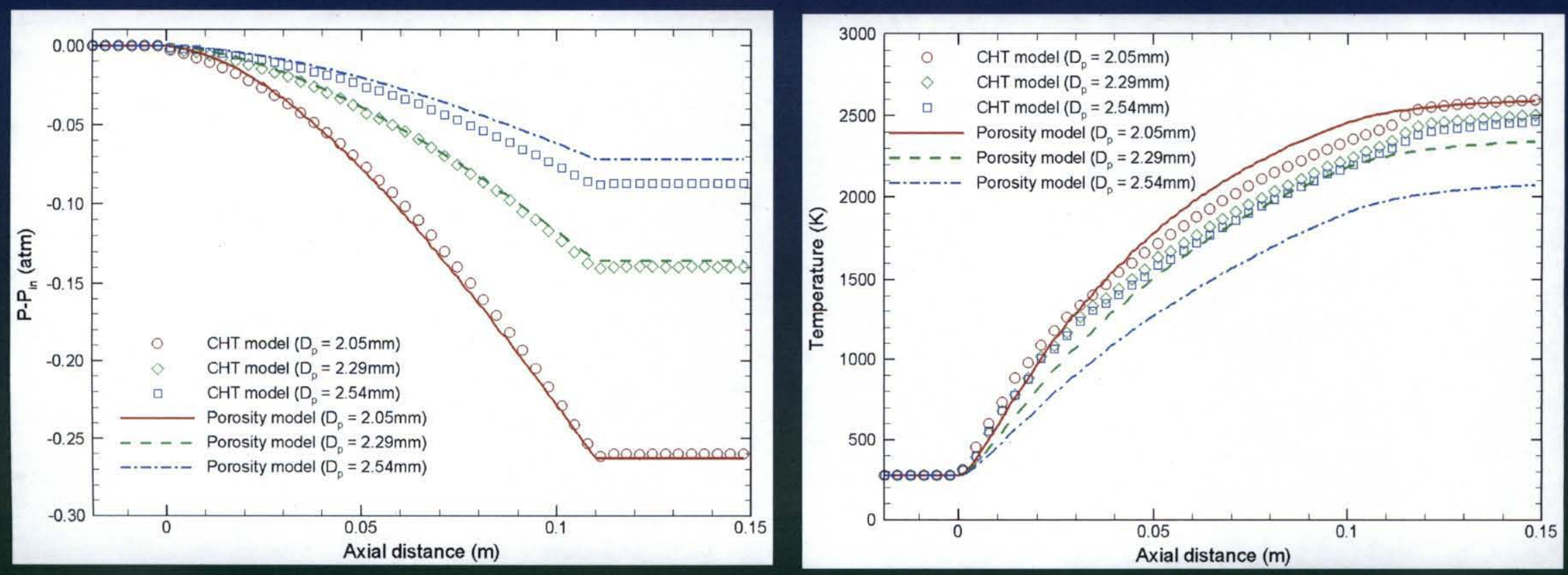


\section{Analysis of Full-Size Flow Element}

- No. of cells: $4.5 \mathrm{M}$, no. of grid points: $98 \mathrm{~K}$

- Geometry and inlet B.C.

\begin{tabular}{|c|c|c|c|c|c|}
\hline \multicolumn{3}{|c|}{ Flow Element Geometry } & \multicolumn{3}{c|}{ Inflow conditions $\left(\mathrm{H}_{2}\right)$} \\
\hline $\begin{array}{c}\text { Element } \\
\text { width }\left(W_{p}\right)\end{array}$ & $\begin{array}{c}\text { Channel } \\
\text { diameter }\left(D_{p}\right)\end{array}$ & $\begin{array}{c}\text { Element } \\
\text { length }\left(L_{p}\right)\end{array}$ & Pressure & Temperature & $\begin{array}{c}\text { Mass flow } \\
\text { rate }\end{array}$ \\
\hline $19.1 \mathrm{~mm}$ & $2.05 \mathrm{~mm}$ & $890 \mathrm{~mm}$ & $39.05 \mathrm{~atm}$ & $279 \mathrm{~K}$ & $0.0147 \mathrm{~kg} / \mathrm{s}$ \\
\hline
\end{tabular}

- Variable thermal properties for flow element and coating layer (listed are reference values at $300 \mathrm{~K}$ )

\begin{tabular}{|l|c|c|c|}
\hline & Thermal conductivity & Density & Specific heat \\
\hline UC-C-ZrC composite & $91 \mathrm{~W} / \mathrm{m} \cdot \mathrm{K}$ & $6142 \mathrm{~kg} / \mathrm{m}^{3}$ & $468.4 \mathrm{~W} \cdot \mathrm{s} / \mathrm{kg} \cdot \mathrm{K}$ \\
\hline ZrC composite & $19 \mathrm{~W} / \mathrm{m} \cdot \mathrm{K}$ & $6531 \mathrm{~kg} / \mathrm{m}^{3}$ & $368 \mathrm{~W} \cdot \mathrm{s} / \mathrm{kg} \cdot \mathrm{K}$ \\
\hline
\end{tabular}




\section{Analysis of Full-Size Flow Element}

- Case \#1: pure cosine profile for power generation

$$
\dot{Q}=\dot{Q}_{\text {max }} \cos \left[\pi\left(x / L_{p}-0.5\right)\right] \quad x: 0 \rightarrow L_{p}
$$

- Case \#2: clipped cosine profile for power generation

$$
\dot{Q}=\dot{Q}_{\text {max }} \min \left\{1,1.5 \cos \left[\pi\left(x / L_{p}-0.5\right)\right]\right\} \quad x: 0 \rightarrow L_{p}
$$

- Total power generated: $491 \mathrm{~kW}$ (80\% designed value) 


\section{Analysis of Full-Size Flow Element}

Case \#1

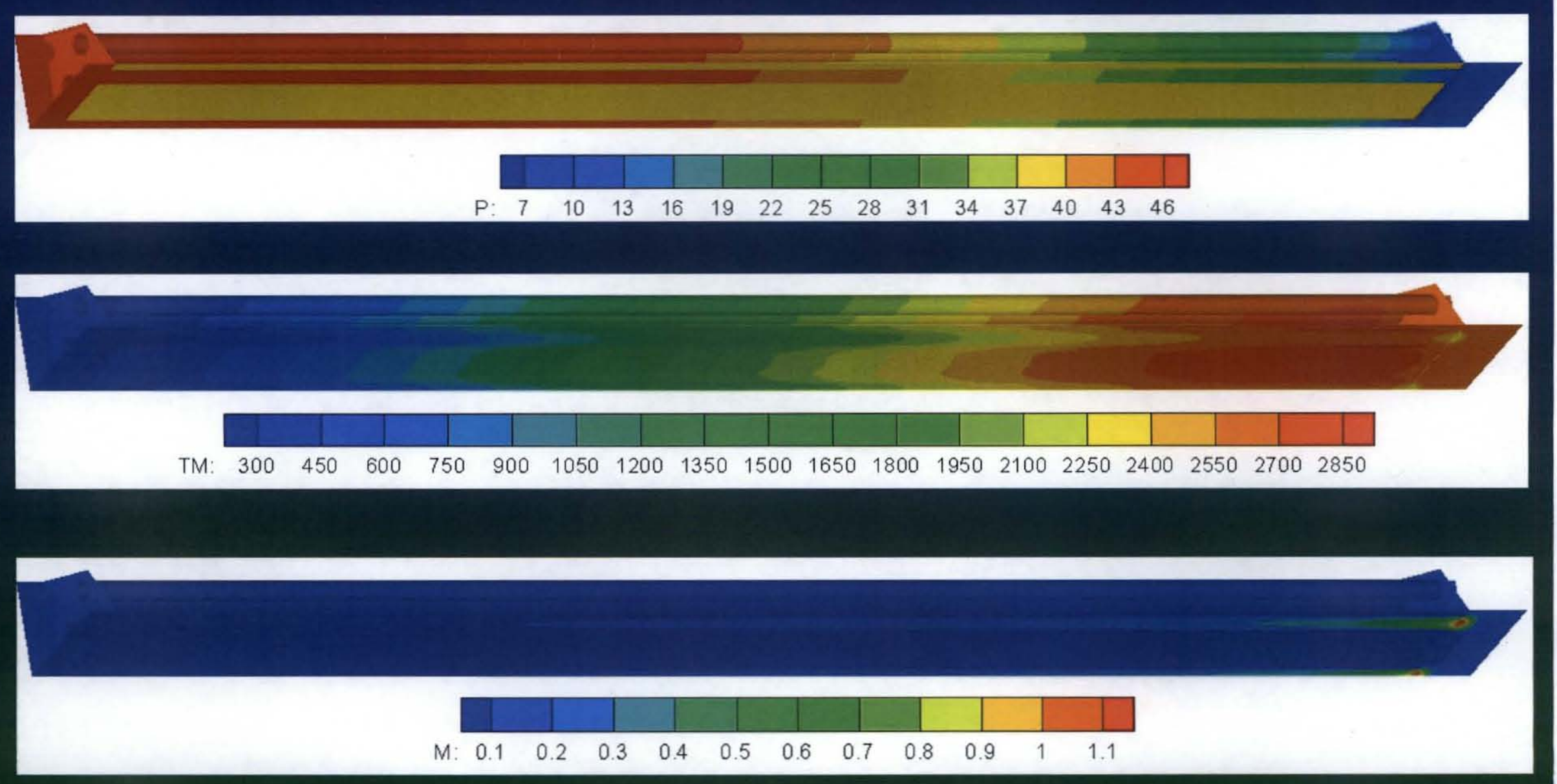




\section{Analysis of Full-Size Flow Element}
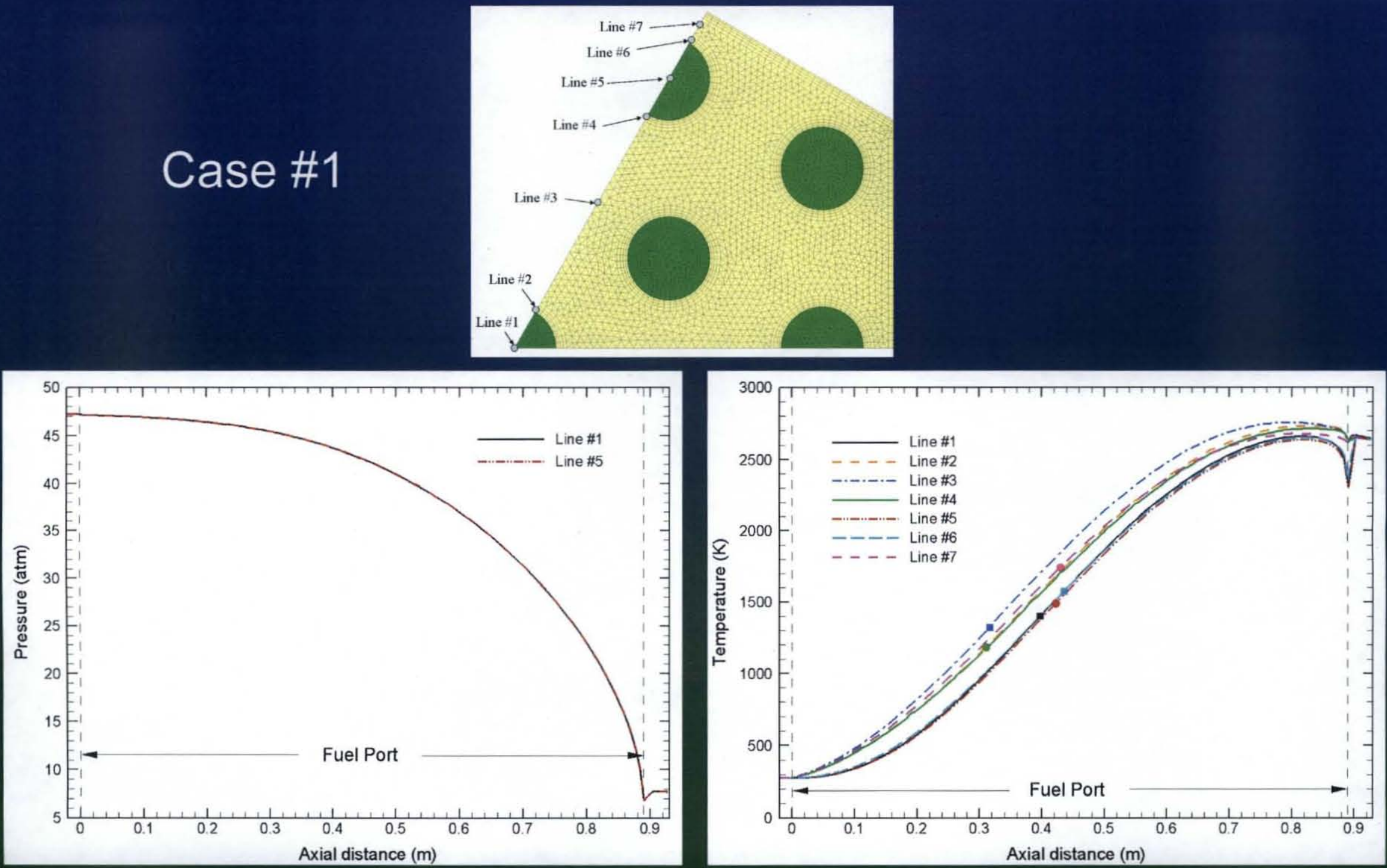


\section{Analysis of Full-Size Flow Element}

\section{Case \#1}

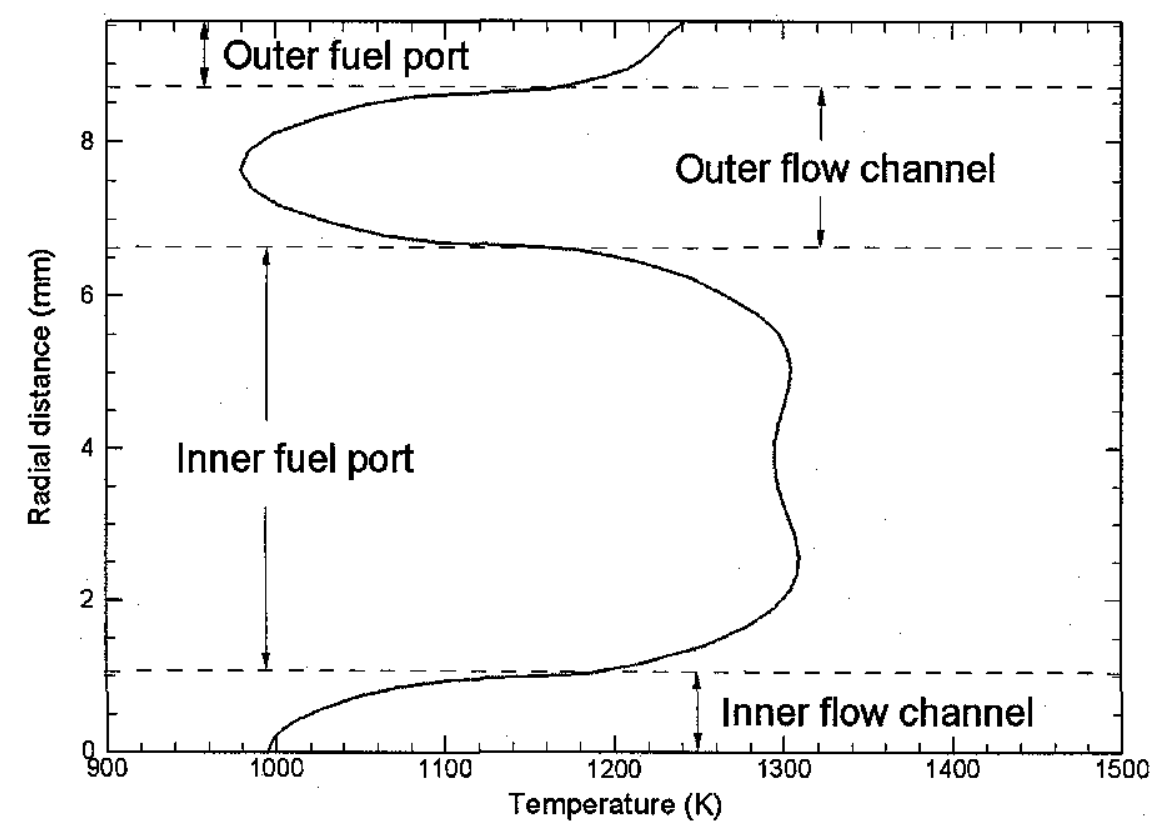

At max. temperature gradient of coating layer

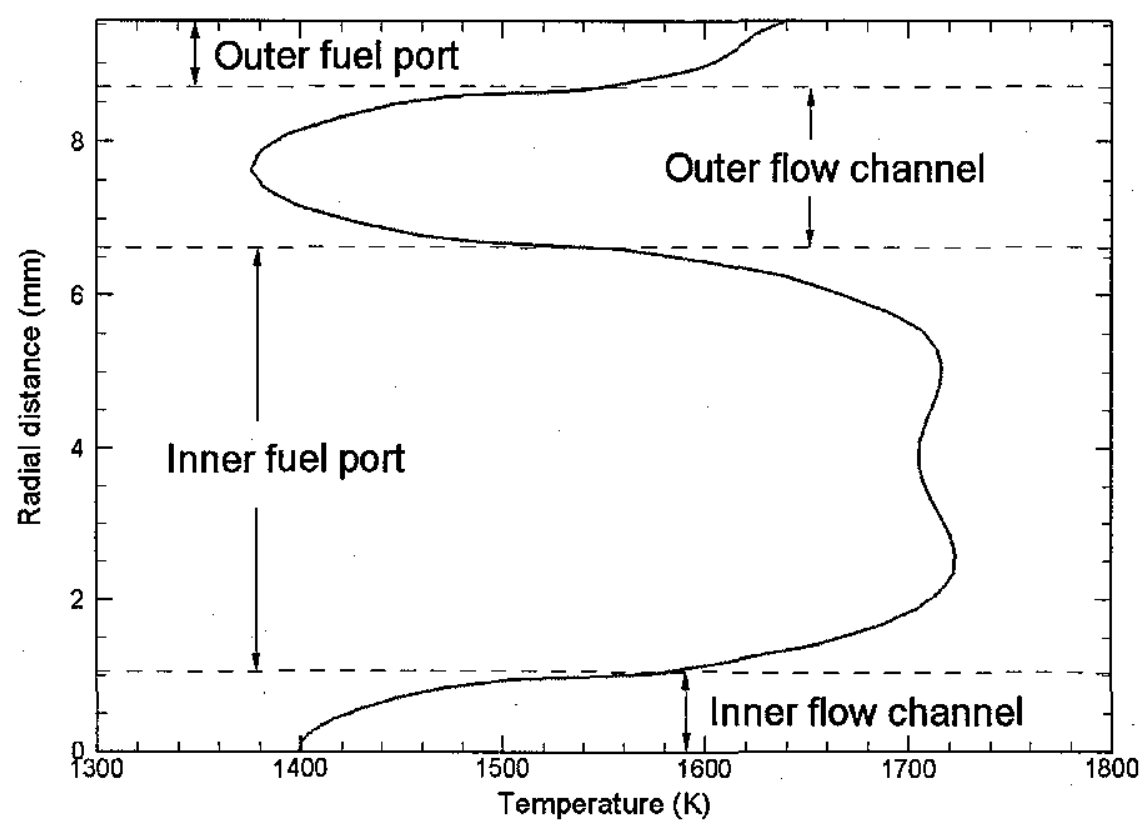

At max. temperature gradient of center flow channel 


\section{Analysis of Full-Size Flow Element}

Case \#2

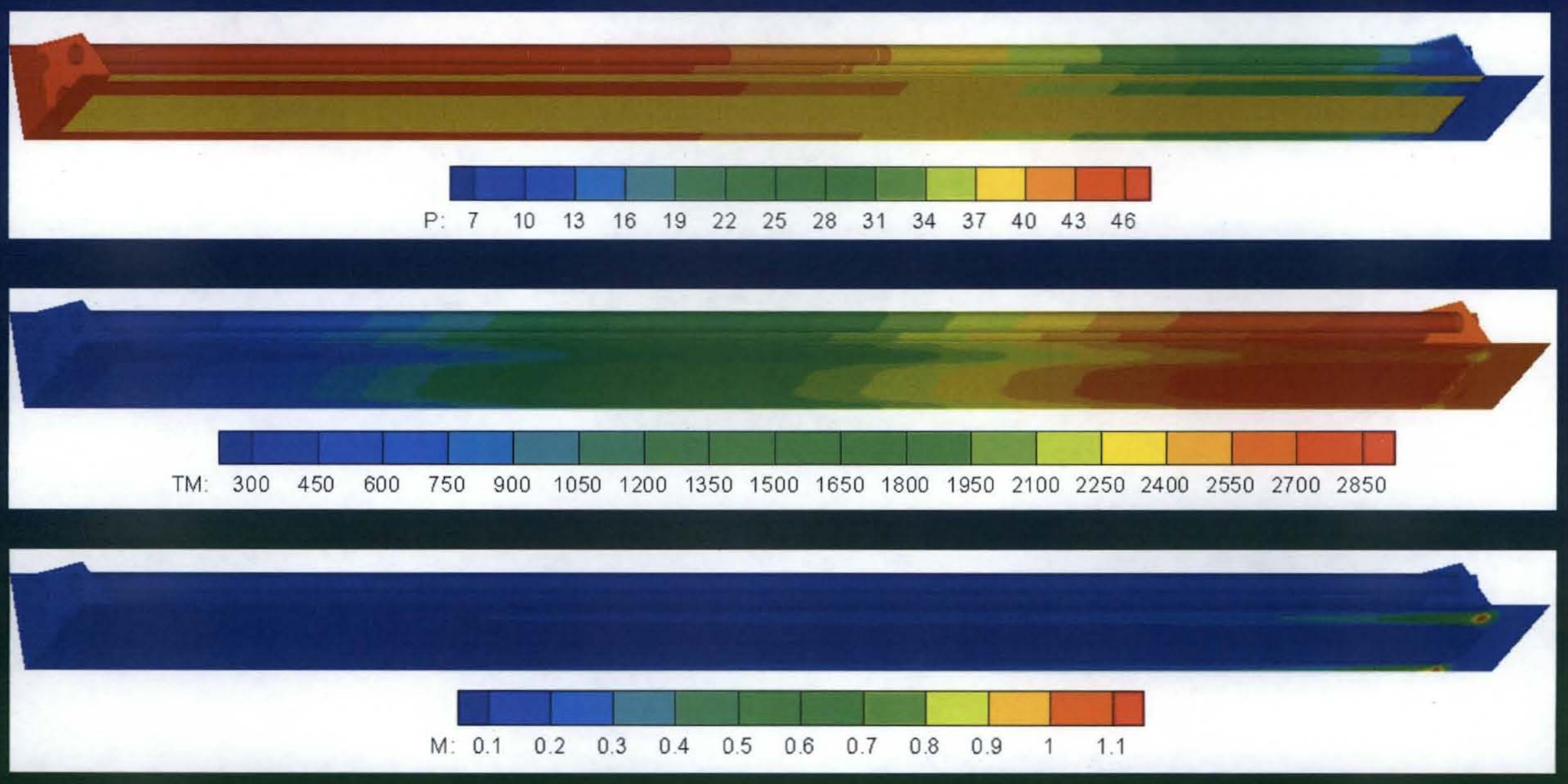




\section{Analysis of Full-Size Flow Element}

Case \#2
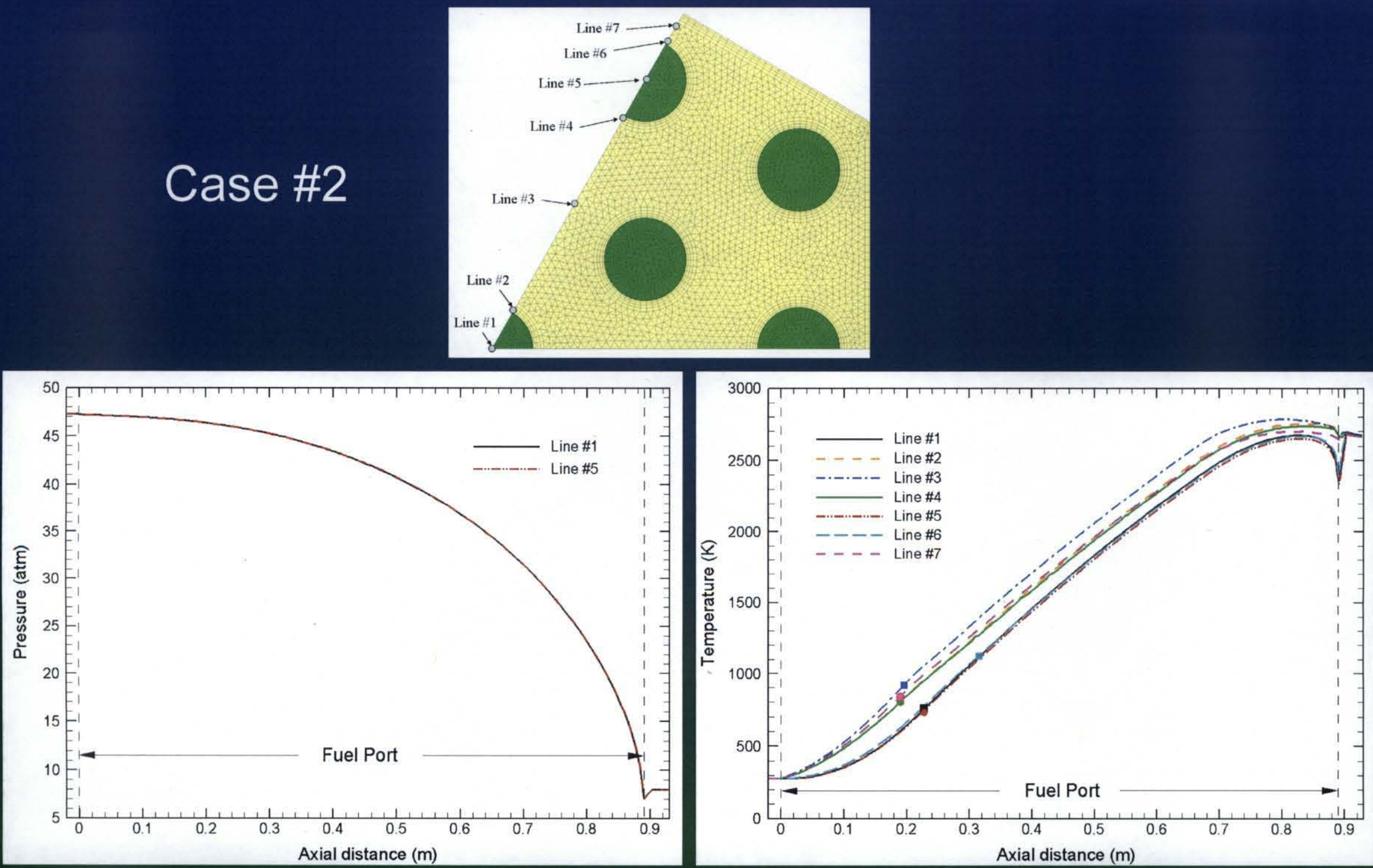


\section{Analysis of Full-Size Flow Element}

\section{Case \#2}

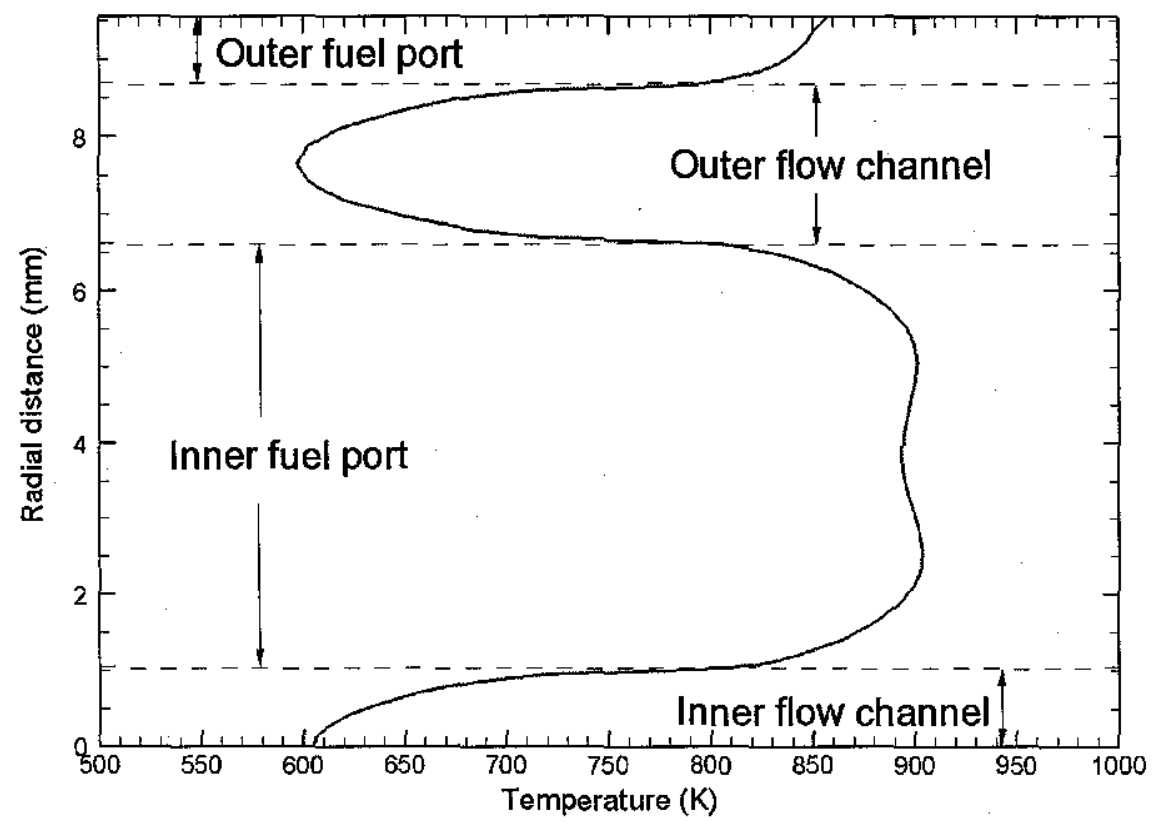

At max. temperature gradient of coating layer

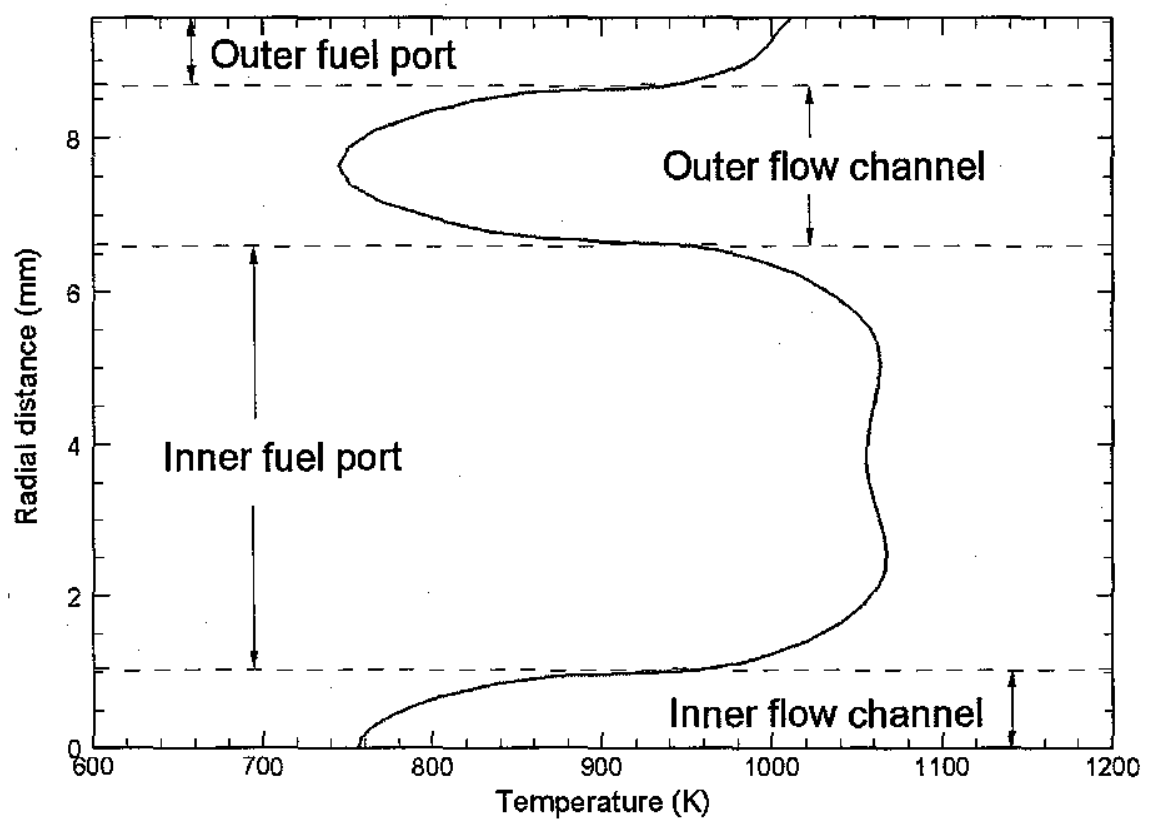

At max. temperature gradient of center flow channel 


\section{CONCLUSIONS}

- The employed CFD model has been successfully applied to simulate thermal-fluid environment of a single flow element

- The use of porosity model can be an efficient numerical approach for simulating an assembly of a huge number of flow elements, but needs further improvement

- The numerical result indicates large thermal gradients at the fluid-coating and coating-fuel interfaces for the selected fuel and coating materials

- Power distribution function can affect the location and level of max. thermal gradient

- Even at $80 \%$ of the designed power level some flow elements possibly have flow chocking in some flow channels 


\section{RECOMMENDATIONS}

- A pie-section of the reactor with a downstream nozzle should be simulated to:

- Account for the multiple flow element effect

- Evaluate the magnitude of max. flow Mach no. in the flow channel with the full power generated

- Calculate distribution of the mass flow rate, temperature and pressure among flow elements

- Conduct thermal stress analysis to examine the possibility of crack of the coating layer

- Different configurations of the flow element (number and area) should be examined to optimize its efficiency

- Neutronic reactions should be included to accurately model power generation 